\title{
FREE MINIMAL ACTIONS OF COUNTABLE GROUPS WITH INVARIANT PROBABILITY MEASURES
}

\author{
GÁBOR ELEK
}

\begin{abstract}
We prove that for any countable group $\Gamma$ there exists a free minimal continuous action $\alpha: \Gamma \curvearrowright \mathcal{C}$ on the Cantor set admitting an invariant Borel probability measure.
\end{abstract}

Keywords. Free minimal actions, uniformly recurrent subgroups, invariant measures

\section{INTRODUCTION}

In [6] Hjorth and Molberg proved that for any countable group $\Gamma$ there exists a free and continuous action of $\Gamma$ on the Cantor set $\mathcal{C}$ that admits an invariant Borel probability measure. Our main goal is to prove that one can also assume the minimality of the action.

Theorem 1. For any countably infinite group $\Gamma$, there exists a free minimal continuous action $\alpha: \Gamma \curvearrowright \mathcal{C}$ on the Cantor set admitting an invariant Borel probability measure.

In fact, we show that $\alpha$ can be chosen to be universal in the following sense.

Theorem 2. There exists a free, minimal action $\alpha: \Gamma \curvearrowright \mathcal{C}$ that satisfies the following condition. Let $\beta: \Gamma \curvearrowright X$ be a free Borel action of the above group $\Gamma$ on the standard Borel space $X$ admitting an invariant Borel probability measure $\mu$. Then there exists a Borel embedding $\Phi: X^{\prime} \rightarrow \mathcal{C}$ commuting with the actions $\alpha$ and $\beta$, such that $X^{\prime}$ is an invariant Borel set of $X$ and $\mu\left(X^{\prime}\right)=1$.

It is important to note that Weiss proved [11] the existence of a topologically free minimal Cantor action satisfying the condition of Theorem 2. Notice however, that topologically free minimal Cantor actions of the free group admitting an invariant measure can be very far from being essentially free. In fact, it is possible that the equivalence relation associated to such topologically free minimal action is hyperfinite [1]. Our approach to prove Theorem 2 is based on the philosophy behind Weiss' proof and the notion of properness.

First we prove a result on Borel universality.

2010 Mathematics Subject Classification. 46L55.

The author was partially supported by the ERC Consolidator Grant "Asymptotic invariants of discrete groups, sparse graphs and locally symmetric spaces" No. 648017. 
Proposition 1.1. For any countably infinite group $\Gamma$ and for any free Borel action $\beta: \Gamma \curvearrowright X$ on the standard Borel space, there exists an injective equivariant Borel map $\Psi_{\beta}^{\prime}: X \rightarrow \operatorname{Free}\left(\mathcal{C}^{\Gamma}\right)$ ( where $\operatorname{Free}\left(\mathcal{C}^{\Gamma}\right)$ is the free part of the Bernoulli $\mathcal{C}$-shift space), such that the closure of the set $\Psi_{\beta}^{\prime}(X)$ is still in Free $\left(\mathcal{C}^{\Gamma}\right)$.

As a consequence, we will obtain the following theorem.

Theorem 3. For any countably infinite group $\Gamma$, there exists a free continuous action $\zeta: \Gamma \curvearrowright \mathcal{C}$ such that for any free Borel action $\beta: \Gamma \curvearrowright X$ on the standard Borel space we have an injective Borel map $\Psi_{\beta}: X \rightarrow \mathcal{C}$ satisfying $\Psi_{\beta} \circ \beta=\zeta \circ \Psi_{\beta}$.

Note that Seward and Tucker-Drob [10] (see also [2]) proved the following result: For any free Borel action $\alpha: \Gamma \curvearrowright X$ there exists a (not necessarily injective) equivariant Borel map $\Psi: X \rightarrow$ Free $\left(\{0,1\}^{\Gamma}\right)$ such that the closure of the set $\Psi(X)$ is still in Free $\left(\{0,1\}^{\Gamma}\right)$.

In 1952 Oxtoby [9] proved that there exists a free, minimal $Z$-action on the Cantor set that is not uniquely ergodic, that is, it admits more than one ergodic invariant Borel probability measures. We will prove the following corollary of Theorem 2.

Corollary 1.1. Any countably infinite group $\Gamma$ has a free, minimal action on the Cantor set that is not uniquely ergodic.

We will also prove a version of Theorem 3 for uniformly recurrent subgroups (Theorem 4) answering a question of Glasner and Weiss in the "universal sense". Finally, we prove Theorem 1. The main idea is to construct an explicit free minimal action for any countably infinite group using an inductive "learning" algorithm. Then we combine this construction with Theorem 3 to obtain our main result.

Acknowledgement: We are grateful to Benjamin Weiss for sending us his paper [11].

\section{The Proof of TheOrem 3}

Let $\Gamma$ be a countable infinite group and $\left\{\sigma_{i}\right\}_{i=1}^{\infty}$ be a generating system of $\Gamma$. Also for $n \geq 1$, let $\Gamma_{n}$ be the subgroup of $\Gamma$ generated by the elements $\left\{\sigma_{i}\right\}_{i=1}^{n}$. We will also assume that if $\Gamma_{n} \neq \Gamma$, then $\sigma_{n+1} \notin \Gamma_{n}$. Hence, if $\Gamma_{n} \neq \Gamma$ then $\Gamma_{n+1} \neq \Gamma_{n}$. Consequently, if $\Gamma_{n}$ is finite, then $\left|\Gamma_{n}\right| \geq 2^{n}$. Let $\alpha: \Gamma \curvearrowright X$ be a Borel action of $\Gamma$ on the standard Borel space $X$. We define a sequence $\left\{\mathcal{G}_{n}\right\}_{n=1}^{\infty}$ of Borel graph structures on $X$ in the following way. If $p, q \in X$, $p \neq q$, then let $(p, q) \in E\left(\mathcal{G}_{n}\right)$ if there exists $1 \leq i \leq n$ such that $\alpha\left(\sigma_{i}\right)(p)=q$ or $\alpha\left(\sigma_{i}\right)(q)=p$. A Borel $\mathcal{C}$-coloring of $X$ is a Borel map $\varphi: X \rightarrow \mathcal{C}$, where $\mathcal{C}=\{0,1\}^{\mathbb{N}}$ is the Cantor set. We say that $\varphi$ is a proper $\mathcal{C}$-coloring with 
respect to $\alpha: \Gamma \curvearrowright X$ if for any $r>0$ there exists $S_{r}>0$ such that for any $p, q \in X(\varphi(p))_{S_{r}} \neq(\varphi(q))_{S_{r}}$, provided that $0<d_{\mathcal{G}_{r}}(p, q) \leq r$, where

- $d_{\mathcal{G}_{r}}$ is the shortest path metric on the components of the Borel graph $\mathcal{G}_{r}$.

- For $\kappa \in \mathcal{C}$ and $s>0,(\kappa)_{s} \in\{0,1\}^{[s]}$, denotes the projection of $\kappa$ onto its first $s$ coordinates. Here $[s]$ denotes the set $\{1,2, \ldots, s\}$.

The Borel coloring $\varphi$ is called separating if for any $p \neq q \in X, \varphi(p) \neq \varphi(q)$.

Lemma 2.1. For any Borel action $\alpha: \Gamma \curvearrowright X$ there exists a separating, proper $\mathcal{C}$-coloring with respect to $\alpha$.

Proof. First, for any $r \geq 1$ we construct a new Borel graph $\mathcal{H}_{r}$ of bounded vertex degree on $X$ such that $(p, q) \in E\left(\mathcal{H}_{r}\right)$ if $0<d_{G_{r}}(p, q) \leq r$. By the classical result of Kechris, Solecki and Todorcevic [7], there exists an integer $m_{r}>0$ and a Borel coloring $\psi_{r}: X \rightarrow\{0,1\}^{\left[m_{r}\right]}$ such that $\psi_{r}(p) \neq \psi_{r}(q)$, whenever $p$ and $q$ are adjacent vertices in the Borel graph $\mathcal{H}_{r}$. Then $\varphi_{1}(p)=$ $\left\{\psi_{1}(p) \psi_{2}(p) \ldots\right\} \in\{0,1\}^{\mathbb{N}}$, defines a proper $\mathcal{C}$-coloring of $X$ with respect to $\alpha$. Now we use the usual trick to obtain a separating coloring. Let $\varphi_{2}: X \rightarrow \mathcal{C}$ be a Borel embedding. Then if $\varphi_{1}(p)=\left\{a_{1} a_{2} a_{3} \ldots\right\}$ and $\varphi_{2}(p)=\left\{b_{1} b_{2} b_{3} \ldots\right\}$ let $\varphi(p)=\left\{a_{1} b_{1} a_{2} b_{2} \ldots\right\}$. Clearly, $\varphi$ is a separating, proper $\mathcal{C}$-coloring with respect to $\alpha$.

Now we prove Proposition 1.1. Let $\alpha: \Gamma \curvearrowright X$ be a free Borel action and let $\varphi: X \rightarrow \mathcal{C}$ be a separating, proper $\mathcal{C}$-coloring. Consider the Bernoulli shift $\mathcal{C}^{\Gamma}$ with the natural left action

$$
L_{\delta}(\rho)(\gamma)=\rho(\gamma \delta) .
$$

The map $\Psi_{\alpha}^{\prime}: X \rightarrow \mathcal{C}^{\Gamma}$ is defined as usual by

$$
\Psi_{\alpha}^{\prime}(x)(\gamma)=\varphi(\alpha(\gamma)(x)) .
$$

Clearly, $\Psi_{\alpha}^{\prime}$ is Borel and $\Gamma$-equivariant and since $\varphi$ is separating, $\Psi_{\alpha}^{\prime}$ is injective as well. Also, $\Psi_{\alpha}^{\prime}(X) \subset \operatorname{Free}\left(\mathcal{C}^{\Gamma}\right)$, where $\operatorname{Free}\left(\mathcal{C}^{\Gamma}\right)$ is the free part of the Bernoulli shift, that is, the set of elements $\rho \in \mathcal{C}^{\Gamma}$ such that if $L_{\delta}(\rho)=\rho$, then $\delta=e_{\Gamma}$. We need to show that if $\rho \in \mathcal{C}^{\Gamma}$ is in the closure of $\Psi_{\alpha}^{\prime}(X)$, then $L_{\gamma}(\rho) \neq \rho$, whenever $\gamma \neq e_{\Gamma}$. It is enough to see that if $\gamma \neq e_{\Gamma}$, then $\rho(\gamma) \neq \rho\left(e_{\Gamma}\right)$. Let $\lim _{n \rightarrow \infty} \Psi_{\alpha}^{\prime}\left(x_{n}\right)=\rho \in \mathcal{C}^{\Gamma}$. Also, let $r>0$ such that

- $\gamma \in \Gamma_{r}$.

- $d_{G_{r}}\left(e_{\Gamma}, \gamma\right) \leq r$, where $d_{G_{r}}$ is the shortest path metric on the left Cayley graph $\operatorname{Cay}\left(\Gamma_{r},\left\{\sigma_{i}\right\}_{i=1}^{r}\right)$.

Thus, for any $n \geq 1$,

$$
d_{G_{r}}\left(\alpha(\gamma)\left(x_{n}\right), x_{n}\right) \leq r
$$

Hence, for any $n \geq 1$,

$$
\left(\varphi\left(\alpha(\gamma)\left(x_{n}\right)\right)\right)_{S_{r}} \neq\left(\varphi\left(x_{n}\right)\right)_{S_{r}}
$$


Since $\rho(\gamma)=\lim _{n \rightarrow \infty} \varphi\left(\alpha(\gamma)\left(x_{n}\right)\right)$ and $\rho\left(e_{\Gamma}\right)=\lim _{n \rightarrow \infty} \varphi\left(x_{n}\right)$, we have that $\rho(\gamma) \neq \rho\left(e_{\Gamma}\right)$. Hence our proposition follows.

Now we prove Theorem 3. Let $\iota: \Gamma \curvearrowright \operatorname{Free}\left(\mathcal{C}^{\Gamma}\right)$ be the natural free action and $\Psi_{\iota}^{\prime}:$ Free $\left(\mathcal{C}^{\Gamma}\right) \rightarrow \operatorname{Free}\left(\mathcal{C}^{\Gamma}\right)$ be the injective $\Gamma$-equivariant Borel map defined in Proposition 1.1. Let $W$ be the closure of $\Psi_{\iota}^{\prime}\left(\operatorname{Free}\left(\mathcal{C}^{\Gamma}\right)\right)$ in $\mathcal{C}^{\Gamma}$. Thus, $W \subset$ Free $\left(\mathcal{C}^{\Gamma}\right)$ is a closed invariant subset. Then, $W$ can be written as the disjoint union of $W_{1}$ and $W_{2}$, where $W_{1}$ is a countable invariant subset and $W_{2}$ is a closed invariant subset homeomorphic to the Cantor set. Indeed, $W$ is an uncountable compact set. Hence $W$ can be written as the disjoint union of $W_{a}$ and $W_{b}$, where $W_{a}$ is homeomorphic to the Cantor set and $W_{b}$ is countable. Clearly, the orbit closure $W_{2}$ of $W_{a}$ has no isolated point and $W_{1}=W \backslash W_{2}$ is a countable invariant set. Now, let $\alpha: \Gamma \curvearrowright X$ be a free Borel action and $\Psi_{\alpha}^{\prime}$ : $X \rightarrow$ Free $\left(\mathcal{C}^{\Gamma}\right)$ be the injective $\Gamma$-equivariant Borel map defined in Proposition 1.1. Let $\tilde{\Psi}_{\alpha}=\Psi_{\iota}^{\prime} \circ \Psi_{\alpha}^{\prime}$. Clearly, $\tilde{\Psi}_{\alpha}: X \rightarrow W$ is an injective $\Gamma$-equivariant Borel map. Now we define the injective $\Gamma$-equivariant Borel map $\Psi_{\alpha}: X \rightarrow W_{2}$ by modifying $\tilde{\Psi}_{\alpha}$ on countably many orbits. Let $Y=\left(\tilde{\Psi}_{\alpha}\right)^{-1}\left(W_{1}\right)$. We can assume that the complement $W_{3}$ of $\tilde{\Psi}_{\alpha}(X)$ in $W_{2}$ is infinite, so we can define an injective $\Gamma$-equivariant map $\Psi^{\prime}: Y \rightarrow W_{3}$. Therefore, we can define the injective $\Gamma$-equivariant Borel map $\Psi_{\alpha}: X \rightarrow W_{2}$ by

- $\Psi_{\alpha}(x)=\tilde{\Psi}_{\alpha}(x)$ if $x \in X \backslash Y$.

- $\Psi_{\alpha}(x)=\Psi^{\prime}(x)$ if $x \in Y$.

Since $W_{2}$ is homeomorphic to $\mathcal{C}$, our theorem follows.

\section{UNIFORMLY RECURRENT SUBGROUPS}

Let $\Gamma$ be a countable group and let $\operatorname{Sub}(\Gamma)$ be the space of subgroups of $\Gamma[5]$. Then $\operatorname{Sub}(\Gamma)$ is a compact, metrizable space and conjugations define a continuous action $c: \Gamma \curvearrowright \operatorname{Sub}(\Gamma)$. Now let $\beta: \Gamma \curvearrowright M$ be a continuous minimal nonfree action of a countable group on a compact metric space. Then we have a natural equivariant map $\operatorname{Stab}_{\beta}: M \rightarrow \operatorname{Sub}(\Gamma)$ from our space $M$ to the compact space of subgroups of $\Gamma$, mapping each point $x \in M$ to its stabilizer subgroup. Glasner and Weiss (Proposition 1.2 [5]) proved that the set $M_{0}$ of the continuity points of the map $\operatorname{Stab}_{\beta}$ is a dense, invariant $G_{\delta}$ subset of $M$ and the closure of $\operatorname{Stab}_{\beta}\left(M_{0}\right)$ in $\operatorname{Sub}(\Gamma)$ is a minimal closed invariant subset of $\operatorname{Sub}(\Gamma)$, that is, a uniformly recurrent subgroup. They asked if for any uniformly recurrent subgroup $Z \subset \operatorname{Sub}(\Gamma)$ there exists a minimal continuous action $\beta: \Gamma \curvearrowright M$ such that $M_{0}=M$ and $\operatorname{Stab}_{\beta}(M)=Z$. This question has been answered in [3] and [8]. Now we show that one can answer the question of Glasner and Weiss in the universal sense.

Theorem 4. Let $\Gamma$ be a countable group and $Z \subset \operatorname{Sub}(\Gamma)$ be a uniformly recurrent subgroup. Then there exists a continuous action $\zeta_{Z}: \Gamma \curvearrowright \mathcal{C}$ such that 
- $\operatorname{Stab}_{\zeta Z}: \mathcal{C} \rightarrow \operatorname{Sub}(\Gamma)$ is continuous everywhere and $\operatorname{Stab}_{{ }_{Z}}(\mathcal{C})=Z$.

- For any Borel action $\alpha: \Gamma \curvearrowright X$ such that for any $x \in X$ the group $\operatorname{Stab}_{\alpha}(x)$ is in $Z$ (we call these actions $(\Gamma, Z)$-actions), there exists an injective Borel map $\Psi_{\alpha}: X \rightarrow \mathcal{C}$ such that $\Psi_{\alpha} \circ \alpha=\zeta_{Z} \circ \Psi_{\alpha}$.

It was proved in Section 5 [3] that there exist countable groups $\Gamma$ and uniformly recurrent subgroups $Z \subset \operatorname{Sub}(\Gamma)$ such that no Borel $(\Gamma, Z)$-action admits an invariant Borel probability measure. However, we have the following nonfree analogue of the aforementioned result of Hjorth and Molberg.

Corollary 3.1. Let $\Gamma$ be a countable group and let $Z \subset \operatorname{Sub}(\Gamma)$ be a uniformly recurrent subgroup. If there exists a Borel $(\Gamma, Z)$-action $\alpha: \Gamma \curvearrowright X$ that admits an invariant Borel probability measure, then there exists a continuous $(\Gamma, Z)$ action $\beta: \Gamma \curvearrowright \mathcal{C}$ on the Cantor set admitting an invariant Borel probability measure such that

- The map $\operatorname{Stab}_{\beta}: \mathcal{C} \rightarrow \operatorname{Sub}(\Gamma)$ is continuous everywhere and

- $\operatorname{Stab}_{\beta}(\mathcal{C})=Z$.

Proof. (of Theorem 4) Let $Z \subset \operatorname{Sub}(\Gamma)$ be a uniformly recurrent subgroup. We define the Bernoulli shift space $\mathcal{C}^{Z}$ of $Z$ in the following way. Let

$$
\mathcal{C}^{Z}=\cup_{H \in Z} \mathcal{F}(H),
$$

where $\mathcal{F}(H)$ is the set of maps $\rho: \Gamma / H \rightarrow \mathcal{C}$ from the left coset space of $H$ to the Cantor set. The action of $\Gamma$ on $\mathcal{C}^{Z}$ is defined as follows.

- If $\rho \in \mathcal{F}(H)$ then $L_{\delta}(\rho) \in \mathcal{F}\left(\delta H \delta^{-1}\right)$ and

- $L_{\delta}(\rho)\left(\gamma \delta H \delta^{-1}\right)=\rho(\gamma \delta H)$.

Lemma 3.1. $L: \Gamma \rightarrow \operatorname{Homeo}\left(\mathcal{C}^{Z}\right)$ is a homomorphism.

Proof. We need to show that if $\rho: \Gamma / H \rightarrow \mathcal{C}$ and $\delta_{1}, \delta_{2} \in \Gamma$, then

$$
L_{\delta_{1}}\left(L_{\delta_{2}}(\rho)\right)=L_{\delta_{1} \delta_{2}}(\rho) \text {. }
$$

Observe that

$$
L_{\delta_{1}}\left(L_{\delta_{2}}(\rho)\right) \in \mathcal{F}\left(\delta_{1} \delta_{2} H \delta_{2}^{-1} \delta_{1}^{-1}\right) \text { and } L_{\delta_{1} \delta_{2}}(\rho) \in \mathcal{F}\left(\delta_{1} \delta_{2} H \delta_{2}^{-1} \delta_{1}^{-1}\right) .
$$

Now

$$
\begin{gathered}
L_{\delta_{1}}\left(L_{\delta_{2}}(\rho)\right)\left(\gamma \delta_{1} \delta_{2} H \delta_{2}^{-1} \delta_{1}^{-1}\right)= \\
=L_{\delta_{2}}(\rho)\left(\gamma \delta_{1} \delta_{2} H \delta_{2}^{-1}\right)=\rho\left(\gamma \delta_{1} \delta_{2} H\right)=L_{\delta_{1} \delta_{2}}(\rho)\left(\gamma \delta_{1} \delta_{2} H \delta_{2}^{-1} \delta_{1}^{-1}\right),
\end{gathered}
$$

hence our lemma follows.

We can equip $\mathcal{C}^{Z}$ with a compact metric structure $d$ such that $\left(\mathcal{C}^{Z}, d\right)$ is homeomorphic to the Cantor set and the $\Gamma$-action above is continuous. Let $\rho_{1}: \Gamma / H_{1} \rightarrow \mathcal{C}, \rho_{2}: \Gamma / H_{2} \rightarrow \mathcal{C}$ be elements of $\mathcal{C}^{Z}$. We say that $\rho_{1}$ and $\rho_{2}$ are $n$-equivalent, $\rho_{1} \equiv_{n} \rho_{2}$ if

- For any $\gamma \in \Gamma, d_{G_{n}}\left(e_{\Gamma}, \gamma\right) \leq n, \gamma \in H_{1}$ if and only if $\gamma \in H_{2}$. 
- For any $\gamma \in \Gamma, d_{G_{n}}\left(e_{\Gamma}, \gamma\right) \leq n$,

$$
\left(\rho_{1}\left(\gamma H_{1}\right)\right)_{n}=\left(\rho_{2}\left(\gamma H_{2}\right)\right)_{n} .
$$

Then we define $d\left(\rho_{1}, \rho_{2}\right):=\frac{1}{2^{n}}$ whenever $\rho_{1} \equiv_{n} \rho_{2}$ and $\rho_{1} \not \equiv_{n+1} \rho_{2}$. Let $\left\{H_{n}\right\}_{n=1}^{\infty}, H \in \operatorname{Sub}(\Gamma), \rho \in \mathcal{F}(H)$ and for any $n \geq 1$ let $\rho_{n} \in \mathcal{F}\left(H_{n}\right)$. Observe that $\left\{\rho_{n}\right\}_{n=1}^{\infty} \rightarrow \rho$ in the $d$-metric if and only if

- $H_{n} \rightarrow H$ in the compact space $\operatorname{Sub}(\Gamma)$ and

- for any $\gamma \in \Gamma, \rho_{n}\left(\gamma H_{n}\right) \rightarrow \rho(\gamma H)$.

We can define $\operatorname{Free}\left(\mathcal{C}^{Z}\right)$ in the usual way. We have that $\rho: \Gamma / H \rightarrow \mathcal{C} \in$ Free $\left(\mathcal{C}^{Z}\right)$ if $L_{\delta}(\rho) \neq \rho$ for any $\delta \notin H$. Clearly, if for any $\delta \notin H, \rho(H) \neq \rho(\delta H)$, then $\rho \in \operatorname{Free}\left(\mathcal{C}^{Z}\right)$. Also, $\operatorname{Stab}_{L}(\rho)=H$. Indeed, if $\delta \in H$, then for any $\gamma \in \Gamma$ we have that

$$
L_{\delta}(\rho)(\gamma H)=\rho(\gamma \delta H)=\rho(\gamma H) .
$$

On the other hand, if $\delta \notin H$, but $\delta H \delta^{-1}=H$, then

$$
L_{\delta}(\rho)(H)=\rho(\delta H) \neq \rho(H) .
$$

Now let $\alpha: \Gamma \curvearrowright X$ be a Borel $(\Gamma, Z)$-action and $\varphi: X \rightarrow \mathcal{C}$ be a separating, proper $\mathcal{C}$-coloring with respect to $\alpha$. We define $\Psi_{\alpha}^{\varphi}: X \rightarrow \mathcal{C}^{Z}$ as follows.

- $\Psi_{\alpha}^{\varphi}(x) \in \mathcal{F}(H)$, where $H=\operatorname{Stab}_{\alpha}(x)$.

- $\Psi_{\alpha}^{\varphi}(x)(\gamma H)=\varphi(\alpha(\gamma)(x))$.

Clearly, $\Psi_{\alpha}^{\varphi}: X \rightarrow \mathcal{C}^{Z}$ is an injective Borel map and $\Psi_{\alpha}^{\varphi}(X) \subset \operatorname{Free}\left(\mathcal{C}^{Z}\right)$.

Lemma 3.2. The map $\Psi_{\alpha}^{\varphi}: X \rightarrow \operatorname{Free}\left(\mathcal{C}^{Z}\right)$ is $\Gamma$-equivariant.

Proof. Let $\delta \in \Gamma$. Then

$$
L_{\delta}\left(\Psi_{\alpha}^{\varphi}(x)\right)\left(\gamma \delta H \delta^{-1}\right)=\Psi_{\alpha}^{\varphi}(x)(\gamma \delta H)=\varphi(\alpha(\gamma \delta)(x)) .
$$

On the other hand,

$$
\Psi_{\alpha}^{\varphi}(\alpha(\delta)(x))\left(\gamma \delta H \delta^{-1}\right)=\varphi(\alpha(\gamma) \alpha(\delta)(x))=\varphi(\alpha(\gamma \delta)(x)) .
$$

Now we prove the nonfree analogue of Proposition 1.1.

Proposition 3.1. For any countably infinite group $\Gamma$ and for any free Borel $(\Gamma, Z)$-action $\alpha: \Gamma \curvearrowright X$, there exists an injective equivariant Borel map $\Psi_{\alpha}^{\prime}: X \rightarrow \operatorname{Free}\left(\mathcal{C}^{Z}\right)$, such that the closure of the set $\Psi_{\alpha}^{\prime}(X)$ is still in $\operatorname{Free}\left(\mathcal{C}^{Z}\right)$.

Proof. Let $\varphi$ and $\Psi_{\alpha}^{\varphi}$ be as above. Let $\left\{x_{n}\right\}_{n=1}^{\infty} \subset X$ such that

$$
\lim _{n \rightarrow \infty} \Psi_{\alpha}^{\varphi}\left(x_{n}\right)=\rho \in \mathcal{F}(H)
$$

and $\delta \notin H$. We need to show that $\rho \in \operatorname{Free}\left(\mathcal{C}^{Z}\right)$. Observe that $\left\{\operatorname{Stab}_{\alpha}\left(x_{n}\right)\right\}_{n=1}^{\infty} \rightarrow H$ in $\operatorname{Sub}(\Gamma)$. Hence, there exists $N>0$ such that $\delta \notin H_{n}$ if $n \geq N$. By properness, there exists $m>0$ such that for all $n \geq N$

$$
\left(\varphi\left(\alpha(\delta)\left(x_{n}\right)\right)\right)_{m} \neq\left(\varphi\left(x_{n}\right)\right)_{m} .
$$

Since, $\lim _{n \rightarrow \infty} \varphi\left(\alpha(\delta)\left(x_{n}\right)\right)=\rho(\delta H)$ and $\lim _{n \rightarrow \infty} \varphi\left(x_{n}\right)=\rho(H)$ we have that $\rho(H) \neq \rho(\delta H)$. Hence, we have that $\rho \in \operatorname{Free}\left(\mathcal{C}^{Z}\right)$. 
Now Theorem 4 follows from Proposition 3.1 exactly the same way as Theorem 1 follows from Proposition 1.1. Note that if $Y \subset \operatorname{Free}\left(\mathcal{C}^{Z}\right)$ is a closed, invariant subset, then the continuity of $\mathrm{Stab}_{L}$ on $Y$ follows from the definition.

\section{Minimal Cantor labelings I.}

The goal of the next three sections is to present an explicit contstruction of free minimal actions for countably infinite groups. Before getting further let us recall some notions from graph theory. Let $G$ be a graph, $S \subset V(G)$ be a subset and $r, s>0$ be integers. Then $S$ is an $s$-net if for any $x \in V(G)$ there exists $y \in S$ such that $d_{G}(x, y) \leqslant s$. Also, $S$ is called an $r$-sparse set if for any $x \neq y \in S, d_{G}(x, y)>r$.

Definition 4.1. $T \subset V(G)$ is an $S$-maximal $r$-sparse set if $T$ is maximal among sets that satisfy the following two properties.

- $T \subseteq S$

- $T$ is $r$-sparse.

Note that an $S$-maximal $r$-sparse set is not necessarily a maximal $r$-sparse set. However, if $T$ is an $S$-maximal $r$-sparse set, then $T$ is also an $S^{\prime}$-maximal $r$-sparse set, provided that $T \subseteq S^{\prime} \subseteq S$.

Lemma 4.1. If $T$ is an $S$-maximal $r$-sparse set, where $S$ is an s-net, then $T$ is an $s+r$-net.

Proof. Let $x \in V(G)$. Then, we have $y \in S$ such that $d_{G}(x, y) \leq s$. By maximality, if $y \notin T$ then there exists $z \in T$ such that $d_{G}(y, z) \leq r$. Hence, $d_{G}(x, T) \leq r+s$.

Now let $\Gamma,\left\{\sigma_{i}\right\}_{i=1}^{\infty},\left\{\Gamma_{r}\right\}_{r=1}^{\infty}$ be as above and for $r \geq 1$ let the graph $G_{r}$ be defined in the following way.

- $V\left(G_{r}\right)=\Gamma$.

- $(p, q) \in E\left(G_{r}\right)$ if for some $1 \leq i \leq r, \sigma_{i} p=q$ or $\sigma_{i} q=p$.

We construct inductively sequence of positive integers

$$
\begin{aligned}
& s_{1}<r_{1}<s_{2}<r_{2}<\ldots, \\
& f(1)<f(2)<f(3)<\ldots
\end{aligned}
$$

and finite sets $\left\{F_{m}\right\}_{m=1}^{\infty}$ with distinguished elements $q_{m} \in F_{m}$ satisfying certain rules. In the following subsection we describe the rules.

4.1. The Rules. We choose the numbers $\left\{s_{i}\right\}_{i=1}^{\infty},\left\{r_{i}\right\}_{i=1}^{\infty},\{f(i)\}_{i=1}^{\infty}$ and $\left\{\left|F_{m}\right|\right\}_{m=1}^{\infty}$ in the following order.

$$
s_{1}, f(1), r_{1},\left|F_{1}\right|, s_{2}, f(2), r_{2},\left|F_{2}\right|, s_{3}, \ldots
$$

Before stating the rules we need a lemma. 
Lemma 4.2. For any $T \geq 1$, there exists $n_{T}>0$ such that if $n_{T} \leq n$, then there is an element $\gamma \in \Gamma_{n}$ so that $d_{G_{n}}\left(\gamma, e_{\Gamma}\right) \geq T$.

Proof. Observe that for all $m \geq 1,\left|B_{T}\left(G_{m}, e_{\Gamma}\right)\right| \leq(3 m)^{T}$. On the other hand, by our definition $\left|\Gamma_{m}\right| \geq 2^{m}$, hence our lemma follows.

Let $s_{1}=10$ and then define $f(1)$ in such a way that

$$
10\left|B_{50}\left(G_{f(1)}, e_{\Gamma}\right)\right|<\left|\Gamma_{f(1)}\right| \text {. }
$$

Note the if $\Gamma_{1}$ is infinite we can define $f(1)$ being equal to 1 . Then, pick $r_{1}$ so that

$$
\left|B_{\frac{1}{10} r_{1}}\left(G_{f(1)}, e_{\Gamma}\right)\right| \geq 10\left|B_{50}\left(G_{f(1)}, e_{\Gamma}\right)\right| .
$$

Finally, choose $\left|F_{1}\right|$ in such a way that $\left|B_{r_{1}}\left(G_{f(1)}, e_{\Gamma}\right)\right|<\left|F_{1}\right|$.

Rule 1. Suppose that $s_{1}, f(1), r_{1},\left|F_{1}\right|, s_{2}, f(2), \ldots,\left|F_{m}\right|$ have already been chosen. Let $\kappa_{m}=\left|F_{m}\right|^{\left|B_{s_{m}}\left(\Gamma_{f(m)}, e_{\Gamma}\right)\right|}$ be the number of labelings of the ball $B_{s_{m}}\left(\Gamma_{f(m)}, e_{\Gamma}\right)$ by the set $F_{m}$. Choose the number $s_{m+1}$ so large (see Lemma 4.3 below) so that for any $n \geq n_{1000 r_{m} \kappa_{m}}$ (see Lemma 4.2) and for any $2 r_{m}$-net $T \subset \Gamma_{n}$ one can pick a subset $L_{T}$ satisfying the following three conditions.

- $\left|L_{T}\right|=\kappa_{m}$

- For any $\delta \in L_{T}$,

$$
\frac{1}{3} s_{m+1} \leq d_{G_{n}}\left(e_{\Gamma}, \delta\right) \leq \frac{2}{3} s_{m+1} .
$$

- For any $\gamma \neq \delta \in L_{T}$,

$$
d_{G_{n}}(\gamma, \delta)>20 r_{m}
$$

Lemma 4.3. The number $s_{m+1}$ can be chosen as it is required above.

Proof. Let $s_{m+1}=1000 r_{m} \kappa_{m}$. Let $c_{1}<c_{2}<\cdots<c_{\kappa_{m}}$ be integers such that for any $1 \leq i \leq \kappa_{m}$ we have $\frac{1}{3} s_{m+1}+10 r_{m} \leq c_{i} \leq \frac{2}{3} s_{m+1}-10 r_{m}$ and $\left|c_{i+1}-c_{i}\right|>100 r_{m}$. Now using Lemma 4.2, we pick elements $\left\{\gamma_{i}\right\}_{i=1}^{\kappa_{m}} \subset \Gamma$ such that $d_{G_{n}}\left(e_{\Gamma}, \gamma_{i}\right)=c_{i}$. For $1 \leq i \leq \kappa_{m}$, let $\delta_{i} \in T$ such that $d_{G_{n}}\left(\gamma_{i}, \delta_{i}\right) \leq 2 r_{m}$. Then $L_{T}:=\left\{\delta_{i}\right\}_{i=1}^{\kappa_{m}}$ satisfies the three conditions in Rule 1 .

Rule 2. Suppose that the numbers $s_{1}, f(1), r_{1},\left|F_{1}\right|, s_{2}, f(2), r_{2}, \ldots, s_{m+1}$ have already been chosen.

Choose $f(m+1)$ in such a way that $f(m+1)>f(m), f(m+1)>n_{1000 r_{m} \kappa_{m}}$ and

$$
10^{m+1}\left|B_{5 s_{m+1}}\left(G_{f(m+1)}, e_{\Gamma}\right)\right|<\left|\Gamma_{f(m+1)}\right| .
$$

Then pick the number $r_{m+1}$ so that both conditions below are satisfied.

- $r_{m+1} \geq 1000\left(\sum_{j=1}^{m+1} 5 r_{j-1}+s_{j}\right)$

- $\left|B_{\frac{1}{10} r_{m+1}}\left(G_{f(m+1)}, e_{\Gamma}\right)\right| \geq 10^{m+1}\left|B_{5 s_{m+1}}\left(G_{f(m+1)}, e_{\Gamma}\right)\right|$. 
Rule 3. Suppose that the numbers $s_{1}, f(1), r_{1},\left|F_{1}\right|, \ldots, r_{m+1}$ have already been chosen. Then pick the number $\left|F_{m+1}\right|$ in such a way that

$$
\left|B_{r_{m+1}}\left(G_{f(m+1)}, e_{\Gamma}\right)\right|<\left|F_{m+1}\right| .
$$

In particular, one has a function $\varphi: \Gamma \rightarrow F_{m}$ such that if $0<d_{G_{f(m)}}(x, y) \leq r_{m}$ then $\varphi(x) \neq \varphi(y)$.

Note that if the group $\Gamma$ is torsion-free then for any $n \geq 1$, we can choose $f(n)=n$. On the other hand, if $\Gamma$ is locally finite, then all the subgroups $\Gamma_{n}$ are finite and we will need to choose all the values $f(n)$ as above.

4.2. Clean labelings. We assume that $\Gamma$ is equipped with a labeling

$$
\Theta=\Theta_{\mathcal{C}} \times \prod_{m=1}^{\infty} \Theta_{m}: \Gamma \rightarrow \mathcal{C} \times \prod_{m=1}^{\infty} F_{m}
$$

satisfying the following conditions.

(1) $\left(\Theta_{1}\right)^{-1}\left(q_{1}\right) \subset \Gamma$ is a maximal $r_{1}$-sparse subset in the graph $G_{1}$.

(2) For any $m>1,\left(\Theta_{m}\right)^{-1}\left(q_{m}\right) \subset \Gamma$ is a $\left(\Theta_{m-1}\right)^{-1}\left(q_{m-1}\right)$-maximal $r_{m}$-sparse subset in the graph $G_{f(m)}$.

(3) For any $m \geq 1, \Theta_{m}(x) \neq \Theta_{m}(y)$ whenever $0<d_{G_{f(m)}}(x, y) \leq r_{m}$.

(4) For any $m \geq 1$,

$$
d_{G_{f(m)}}\left(e_{\Gamma},\left(\Theta_{m}\right)^{-1}\left(q_{m}\right)\right)>10 s_{m} .
$$

We call such a labeling a clean labeling of $\Gamma$. It is easy to see that such clean labelings exist using Rule 3. In Section 9 we will call a labeling almost clean labeling if it satisfies the first three conditions above. From now on we use $\mathcal{F}$ as a shorthand for $\mathcal{C} \times \prod_{m=1}^{\infty} F_{m}$. Let $\Theta^{\prime}=\Theta_{\mathcal{C}}^{\prime} \times \prod_{m=1}^{\infty} \Theta_{m}^{\prime}: \Gamma \rightarrow \mathcal{F}$ be a clean labeling and $j \geq 1$ be an integer. Let $\mathcal{F}_{j}=\{0,1\}^{\{1,2, \ldots, j\}} \times \prod_{m=1}^{j} F_{j}$, let $z \in \Gamma$ and $t>1$ be an integer. Then $B_{t}^{\Theta^{\prime}, j}\left(G_{f(j)}, z\right)$ is a $\mathcal{F}_{j}$-labeled $G_{f(j)}$-ball around $z$ of radius $t$, where the label of $y \in B_{t}\left(G_{f(j)}, z\right)$ is given as

$$
\Theta_{[j]}^{\prime}(y)=\left(\Theta_{\mathcal{C}}^{\prime}(y)\right)_{j} \times \prod_{m=1}^{j} \Theta_{m}^{\prime}(y) .
$$

4.3. Patchings. Patching is an elementary construction that turns one clean labeling into another clean labeling using a third clean labeling. First, we define "regular $n$-patchings". Suppose that two clean labelings $\Theta^{a}=\Theta_{\mathcal{C}}^{a} \times$ $\prod_{m=1}^{\infty} \Theta_{m}^{a}: \Gamma \rightarrow \mathcal{F}$ and $\Theta^{d}=\Theta_{\mathcal{C}}^{d} \times \prod_{m=1}^{\infty} \Theta_{m}^{d}: \Gamma \rightarrow \mathcal{F}$ have already been given. Let $x \in \Gamma$ such that $\Theta_{n}^{d}(x)=q_{n}$. Consider the $\mathcal{F}_{n}$-labeled $G_{f(n)}$-ball $B=B_{s_{n}+r_{n-1}}^{\Theta^{d}, n}\left(G_{f(n)}, x\right)$. The ball is the patch we wish to insert into $\Gamma$ in order to turn $\Theta^{a}$ into the new clean labeling $\Theta^{b}$. In the course of the paper we will refer to the change of labeling described in the next proposition as "patching the $\mathcal{F}_{n}$-labeled $G_{f(n)}$-ball $B_{s_{n}+r_{n-1}}^{\Theta^{d}, n}\left(G_{f(n)}, x\right)$ into $\Theta^{a}$ around the vertex $y$." 
Proposition 4.1. Let $y \in \Gamma$ such that $\Theta_{n}^{a}(y)=q_{n}$. Then, there exists a clean labeling $\Theta^{b}=\Theta_{\mathcal{C}}^{b} \times \prod_{m=1}^{\infty} \Theta_{m}^{b}: \Gamma \rightarrow \mathcal{F}$ such that

- For any $z \in \Gamma,\left(\Theta_{\mathcal{C}}^{b}\right)_{n^{+}}=\left(\Theta_{\mathcal{C}}^{a}\right)_{n^{+}}$, where for $c \in\{0,1\}^{\mathbb{N}},(c)_{n^{+}}$denotes the projection of $c$ onto $\{0,1\}^{\mathbb{N} \backslash\{1,2, \ldots, n\}}$.

- For any $z \in \Gamma$ such that $d_{G_{f(n)}}(y, z) \geq s_{n}+4 r_{n-1}$, we have $\Theta_{\mathcal{C}}^{b}(z)=$ $\Theta_{\mathcal{C}}^{a}(z)$ (in particular, $\Theta_{\mathcal{C}}^{b}(z)=\Theta_{\mathcal{C}}^{a}(z)$, if $z$ is not in the $\Gamma_{f(n) \text {-orbit }}$ of $y)$.

- For any $z \in \Gamma$ such that $d_{G_{f(n)}}(y, z) \leq s_{n}+r_{n-1}$, we have $\left(\Theta_{\mathcal{C}}^{b}(z)\right)_{n}=$ $\left(\Theta_{\mathcal{C}}^{d}\left(z y^{-1} x\right)_{n}\right.$

- For $m \geq n+1$, we have $\Theta_{m}^{b}=\Theta_{m}^{a}$.

- For $m \leq n$ and $z \in \Gamma$ such that $d_{G_{f(n)}}(y, z) \geq s_{n}+4 r_{n-1}$, we have $\Theta_{m}^{b}(z)=\Theta_{m}^{a}(z)$ (again, $\Theta_{m}^{b}(z)=\Theta_{m}^{a}(z)$, if $z$ is not in the $\Gamma_{f(n)}$-orbit of $y$ ).

- For $m \leq n$ and $z \in \Gamma$ such that $d_{G_{f(n)}}(y, z) \leq s_{n}+r_{n-1}$, we have $\Theta_{m}^{b}(z)=\Theta_{m}^{d}\left(z y^{-1} x\right)$.

Proof. We need to define only $\Theta_{m}^{b}: \Gamma \rightarrow F_{m}$ for each $m \leq n-1$. In the first round we will define $\left(\Theta_{m}^{b}\right)^{-1}\left(q_{m}\right)$ for each $m$, in the second (much easier) round we will define the remaining part of the maps $\Theta_{m}^{b}$. For $1 \leq i \leq n-1$, let

$$
\kappa_{i} \subset B_{s_{n}+2 r_{n-1}}\left(G_{f(n)}, y\right) \cup\left(\Gamma \backslash B_{s_{n}+3 r_{n-1}}\left(G_{f(n)}, y\right)\right)
$$

be defined in the following way.

- If $d_{G_{f(n)}}(z, y)>s_{n}+3 r_{n-1}$ then let $z \in \kappa_{i}$ if and only if $\Theta_{i}^{a}(z)=q_{i}$.

- If $d_{G_{f(n)}}(z, y) \leq s_{n}+2 r_{n-1}$, then let $z \in \kappa_{i}$ if and only if $\Theta_{i}^{d}\left(z y^{-1} x\right)=$ $q_{i}$.

We also define $\kappa_{0}$ as the empty set. One can observe that $\kappa_{n-1} \subset \kappa_{n-2} \subset$ $\cdots \subset \kappa_{1}$ and for any $1 \leq i \leq n-1$, the set $\kappa_{i}$ is $r_{i}$-sparse in $G_{f(i)}$. Let $\kappa_{1} \subset \lambda_{1}$ be an arbitrary maximal $r_{1}$-sparse set. Now, let $\kappa_{2} \subset \lambda_{2}$ be a $\lambda_{1}$-maximal $r_{2}$-sparse set. Inductively, we construct the sets

$$
\lambda_{n-1} \subset \lambda_{n-2} \subset \cdots \subset \lambda_{1}
$$

so that for any $1 \leq i \leq n-1$ we have $\kappa_{i} \subset \lambda_{i}$ and $\lambda_{i}$ is a $\lambda_{i-1}$-maximal $r_{i}$-sparse set.

Lemma 4.4. Let $1 \leq i \leq n-1$ and $z_{i} \in B_{s_{n}+r_{n-1}}\left(G_{f(n)}, y\right)$. Also, let $z_{i} \in \lambda_{i}$. Then $z_{i} \in \kappa_{i}$. Similarly, if $z_{i} \in \Gamma \backslash B_{s_{n}+4 r_{n-1}}\left(G_{f(n)}, y\right)$ and $z_{i} \in \lambda_{i}$, then $z_{i} \in \kappa_{i}$.

Proof. Suppose that $z_{1} \in B_{s_{n}+r_{n-1}}\left(G_{f(n)}, y\right)$ such that $z_{1} \in \lambda_{1} \backslash \kappa_{1}$.

Since $\left(\Theta_{1}^{d}\right)^{-1}\left(q_{1}\right)$ is a maximal $r_{1}$-sparse set, there exists $w_{1} \in\left(\Theta_{1}^{d}\right)^{-1}\left(q_{1}\right)$ such that $d_{G_{f(1)}}\left(z_{1} y^{-1} x, w_{1}\right) \leq r_{1}$. That is, $d_{G_{f(1)}}\left(z_{1}, w_{1} x^{-1} y\right) \leq r_{1}$ and

$d_{G_{f(1)}}\left(y, w_{1} x^{-1} y\right) \leq s_{n}+2 r_{n-1}$. Thus, $w_{1} x^{-1} y \in \kappa_{1} \subset \lambda_{1}$ leading to a contradiction. Suppose that our statement holds for all $1 \leq k \leq i$. Let $z_{i+1} \in$ $B_{s_{n}+r_{n-1}}$ and $z_{i+1} \in \lambda_{i+1} \backslash \kappa_{i+1}$. By the induction argument, $z_{i+1} \in \kappa_{i}$. Since $\left(\Theta_{i+1}^{d}\right)^{-1}\left(q_{i+1}\right)$ is a $\left(\Theta_{i}^{d}\right)^{-1}\left(q_{i}\right)$-maximal $r_{i+1}$-sparse set, there exists $w_{i+1} \in$ 
$\left(\Theta_{i+1}^{d}\right)^{-1}\left(q_{i+1}\right)$ such that $d_{G_{i+1}}\left(z_{i+1} y^{-1} x, w_{i+1}\right) \leq r_{i+1}$. That is, $d_{G_{i+1}}\left(z_{i+1}, w_{i+1} x^{-1} y\right) \leq r_{i+1}$ and $d_{G_{i+1}}\left(y, w_{i+1} x^{-1} y\right) \leq s_{n}+2 r_{n-1}$. Therefore, $w_{i+1} x^{-1} y \in \lambda_{i+1}$ in contradiction with the fact that $\lambda_{i+1}$ is $r_{i+1}$-sparse. The proof of the second part can be done similarly.

Now, for $1 \leq i \leq n-1$ let

- $\Theta_{i}^{b}(z)=q_{i}$ if $z \in \lambda_{i}$.

- $\Theta_{i}^{b}(z)=\Theta_{i}^{d}\left(z y^{-1} x\right)$ if $z \in B_{s_{n}+r_{n-1}}\left(G_{f(n)}, y\right)$.

- $\Theta_{i}^{b}(z)=\Theta_{i}^{a}(z)$ if $z \in\left(\Gamma \backslash B_{s_{n}+4 r_{n-1}}\left(G_{f(n)}, y\right)\right)$.

Also, let $\Theta_{n}^{b}(z)=\Theta_{n}^{d}\left(z y^{-1} x\right)$ if $z \in B_{s_{n}+r_{n-1}}\left(G_{f(n)}, y\right)$ and $\Theta_{n}^{b}(z)=\Theta_{n}^{a}(z)$ if $z \in\left(\Gamma \backslash B_{s_{n}+4 r_{n-1}}\left(G_{f(n)}, y\right)\right)$. Then for $1 \leq i \leq n$, let us extend $\Theta_{i}^{b}$ onto the set

$$
\left.\left\{z \mid s_{n}+r_{n-1}<d_{G_{f(n)}}(y, z) \leq s_{n}+4 r_{n-1}\right)\right\} \backslash\left(\Theta_{i}^{b}\right)^{-1}\left(q_{i}\right)
$$

to obtain a clean labeling. Note that $1_{\Gamma} \notin B_{s_{n}+5 r_{n-1}}\left(G_{f(n)}, y\right)$, hence by Rule 2 such extensions $\Theta_{i}^{b}$ clearly exist.

We need another kind of patching in our construction that we call "supersize $n$-patchings". Let $x \in \Gamma$ such that $\Theta_{n}^{d}(x)=q_{n}$. Consider the $\mathcal{F}_{n}$-labeled $G_{f(n)^{-}}$ ball $B=B_{3 r_{n}}^{\Theta^{d}, n}\left(G_{f(n)}, x\right)$. The following proposition is about how to insert $B$ into $\Theta^{a}$. Notice that in the case of regular patchings the ball $B$ we use as a patch contained only one element of $\left(\Theta_{n}^{d}\right)^{-1}\left(q_{n}\right)$. In the case of supersize patchings the ball $B$ contains many elements of $\left(\Theta_{n}^{d}\right)^{-1}\left(q_{n}\right)$. Nevertheless, $B$ will not contain any element of the set $\left(\Theta_{n+1}^{d}\right)^{-1}\left(q_{n+1}\right)$. The next proposition is the "supersized" version of Proposition 4.1.

Proposition 4.2. Let $y \in \Gamma$ such that $\Theta_{n}^{a}(y)=q_{n}$ and $d_{G}\left(y,\left(\Theta_{n+1}^{a}\right)^{-1}\left(q_{n+1}\right)\right)>20 r_{n}$. Then, there exists a clean labeling $\Theta^{b}=\Theta_{\mathcal{C}}^{b} \times$ $\prod_{m=1}^{\infty} \Theta_{m}^{b}: \Gamma \rightarrow \mathcal{F}$ such that

- For any $z \in \Gamma,\left(\Theta_{\mathcal{C}}^{b}\right)_{n^{+}}=\left(\Theta_{\mathcal{C}}^{a}\right)_{n^{+}}$.

- For any $z \in \Gamma$ such that $d_{G}(y, z) \geq 7 r_{n}$, we have $\Theta_{\mathcal{C}}^{b}(z)=\Theta_{\mathcal{C}}^{a}(z)$.

- For any $z \in \Gamma$ such that $d_{G}(y, z) \leq 3 r_{n}$, we have $\left(\Theta_{\mathcal{C}}^{b}(z)\right)_{n}=\left(\Theta_{\mathcal{C}}^{d}\left(z y^{-1} x\right)\right)_{n}$.

- For $m \geq n+1$, let $\Theta_{m}^{b}=\Theta_{m}^{a}$.

- For $m \leq n$ and $z \in \Gamma$ such that $d_{G_{f(n)}}(y, z) \geq 7 r_{n}$, we have $\Theta_{m}^{b}(z)=$ $\Theta_{m}^{a}(z)$

- For $m \leq n$ and $z \in \Gamma$ such that $d_{G_{f(n)}}(y, z) \leq 3 r_{n}$, we have $\Theta_{m}^{b}(z)=$ $\Theta_{m}^{d}\left(z y^{-1} x\right)$.

Proof. First we construct sets $\lambda_{n} \subset \lambda_{n-1} \subset \cdots \subset \lambda_{1}$, where $\kappa_{i} \subset \lambda_{i}$ for any $1 \leq i \leq n, \lambda_{1}$ is a maximal $r_{1}$-sparse set and if $i>1$, then $\lambda_{i}$ is a $\lambda_{i-1^{-}}$ maximal $r_{i}$-sparse set. Then we can proceed in the same way as in the proof of Proposition 4.1. 


\section{Minimal Cantor labelings II. (COdeballs)}

Let $\Theta^{\prime}=\Theta_{\mathcal{C}}^{\prime} \times \prod_{m=1}^{\infty} \Theta_{m}^{\prime}: \Gamma \rightarrow \mathcal{F}$ and $\Theta^{\prime \prime}=\Theta_{\mathcal{C}}^{\prime \prime} \times \prod_{m=1}^{\infty} \Theta_{m}^{\prime \prime}: \Gamma \rightarrow \mathcal{F}$ be clean labelings. Let $\rho>0$ be an integer. We say that the $\mathcal{F}_{j}$-labeled $G_{f(j)}$-ball $B_{t}^{\Theta^{\prime}, j}\left(G_{f(j)}, z\right)$ is $(\rho, j)$-syndetic in $\Theta^{\prime \prime}$ if for any $g \in \Gamma$ there exists $h \in \Gamma$ such that

- $d_{G_{f(j)}}(g, h) \leq \rho$.

- $B_{t}^{\Theta^{\prime}, j}\left(G_{f(j)}, z\right)$ is isomorphic (as $\mathcal{F}_{j}$-labeled $G_{f(j)}$-balls) to $B_{t}^{\Theta^{\prime \prime}, j}\left(G_{f(j)}, h\right)$, $B_{t}^{\Theta^{\prime}, j}\left(G_{f(j)}, z\right) \cong B_{t}^{\Theta^{\prime \prime}, j}\left(G_{f(j)}, h\right)$, that is for any $y \in B_{t}\left(G_{f(j)}, z\right)$ we have that

$$
\Theta_{[j]}^{\prime}(y)=\Theta_{[j]}^{\prime \prime}\left(y z^{-1} h\right)
$$

Definition 5.1. Let $\Theta^{\prime}$ and $\Theta^{\prime \prime}$ be labelings as above. We say that the $\mathcal{F}_{j^{-}}$ labeled $G_{f(j)}$-ball $B_{l}^{\Theta^{\prime \prime}, j}\left(G_{f(j)}, x\right)$ fully contains an isomorphic copy of the $G_{f(j)}$-ball $B_{l^{\prime}}^{\Theta^{\prime}, j}\left(G_{f(j)}, x^{\prime}\right)$ if there exists $w \in \Gamma, d_{G_{f(j)}}(x, w)+l^{\prime}<l$ such that $B_{l^{\prime}}^{\Theta^{\prime}, j}\left(G_{f(j)}, x^{\prime}\right)$ is isomorphic to $B_{l^{\prime}}^{\Theta^{\prime \prime}, j}\left(G_{f(j)}, w\right)$ as $\mathcal{F}_{j}$-labeled $G_{f(j)}$-balls.

We will start with a clean labeling $\Theta$ and inductively construct a sequence of clean labelings $\left\{\Theta^{i}\right\}_{i=1}^{\infty}$ such that $\Theta^{1}=\Theta$ and for any $z \in \Gamma$ there exists an integer $i_{z}>0$ such that if $i \geq i_{z}$ then $\Theta^{i}(z)=\Theta^{i_{z}}(z)$. Hence, for each $z \in \Gamma$, $\lim _{i \rightarrow \infty} \Theta^{i}(z)=\Theta^{\infty}(z)$ exists. The clean labeling $\Theta^{\infty}: \Gamma \rightarrow \mathcal{F}$ will generate a free, minimal subshift in the Bernoulli shift space $\mathcal{F}^{\Gamma}$.

The construction of $\Theta^{2}$. Our input is the clean labeling $\Theta=\Theta^{1}$. Pick any $z_{2} \in \Gamma$ such that $\Theta_{2}^{1}\left(z_{2}\right)=q_{2}$. First we modify the labeling $\Theta^{1}$ on the $G_{f(2)^{-}}$ ball $B_{s_{2}}\left(G_{f(2)}, z_{2}\right)$. Let $\mathcal{A}^{1}=\left\{A_{i}^{1}\right\}_{i=1}^{\tau_{1}}$ be the set of all $\mathcal{F}_{1}$-labeled $G_{f(1)}$-balls of radius $s_{1}$ in $\Theta^{1}$ up to isomorphism. Let $\left\{w_{i}^{1}\right\}_{i=1}^{\tau_{1}} \subset B_{s_{2}}\left(G_{f(2)}, z_{2}\right) \cap\left(\Theta_{1}^{1}\right)^{-1}\left(q_{1}\right)$ be a subset of vertices such that

- For any $1 \leq i \leq \tau_{1}$,

$$
\frac{1}{3} s_{2} \leq d_{G_{f(2)}}\left(z_{2}, w_{i}^{1}\right) \leq \frac{2}{3} s_{2} .
$$

- For any $1 \leq i \neq j \leq \tau_{1}$,

$$
d_{G_{f(2)}}\left(w_{i}^{1}, w_{j}^{1}\right)>20 r_{1} .
$$

Then for each $1 \leq i \leq \tau_{1}$ pick $z_{i}^{1} \in\left(\Theta_{1}^{1}\right)^{-1}\left(q_{1}\right)$ such that $B_{2 r_{1}}^{\Theta^{1}}\left(G_{f(1)}, z_{i}^{1}\right)$ fully contains an isomorphic copy of the $G_{f(1)}$-ball $A_{i}^{1}$. Then using Proposition 4.2 , for each $1 \leq i \leq \tau_{1}$ let us simultaneously patch the balls $B_{3 r_{1}}^{\Theta^{1}, 1}\left(G_{f(1)}, z_{i}^{1}\right)$ into $\Theta^{1}$ around $w_{i}^{1}$. Since $\left(\Theta_{1}^{1}\right)^{-1}\left(q_{1}\right)$ is a maximal $r_{1}$-sparse set such a family of vertices $\left\{z_{i}^{1}\right\}_{i=1}^{\tau_{1}}$ indeed exists. Hence, we obtain a modified clean labeling $\hat{\Theta}^{2}: \Gamma \rightarrow \mathcal{F}$. We call the $\mathcal{F}_{2}$-labeled $G_{f(2)}$-ball $B_{2}=B_{s_{2}}^{\hat{\Theta}^{2}, 2}\left(G_{f(2)}, z_{2}\right)$ the 2-codeball. We finish the construction of $\Theta^{2}$ by patching 
the ball $B_{s_{2}+r_{1}}^{\hat{\Theta}^{2}, 2}\left(G_{f(2)}, z_{2}\right)$ simultaneously into $\Theta^{1}$ centered around all vertices $y \in\left(\Theta_{2}^{1}\right)^{-1}\left(q_{2}\right)$, using Proposition 4.1. Since $\left(\Theta_{1}^{1}\right)^{-1}\left(q_{1}\right)$ is an $r_{1}$-net and $\left(\Theta_{2}^{1}\right)^{-1}\left(q_{2}\right)$ is a $\left(\Theta_{1}^{1}\right)^{-1}\left(q_{1}\right)$-maximal $r_{2}$-sparse net, by Lemma 4.1 each ball $A_{j}^{1} \in$ $\mathcal{A}^{1}$ is $\left(2 r_{2}, 2\right)$-syndetic in $\Theta^{2}$. Note that the 2-codeballs are the analogues of the 2 -welcome words of [11]. We will see that for any $i \geq 1$ and $x \in\left(\Theta_{2}^{i}\right)^{-1}\left(q_{2}\right)$,

$$
B_{s_{2}}^{\Theta^{i}, 2}\left(G_{f(2)}, x\right) \cong B_{2} .
$$

Therefore, all balls $A_{j}^{1} \in \mathcal{A}^{1}$ remain $\left(2 r_{2}, 2\right)$-syndetic in the process. It is important to note that it is possible that the set of $\mathcal{F}_{1}$-labeled $G_{f(1)}$-balls of radius $r_{1}$ will increase when we turn the clean labeling $\Theta^{1}$ into $\Theta^{2}$.

The construction of $\Theta^{3}$. Our input is now the clean labeling $\Theta^{2}$. Pick any $z_{3} \in \Gamma$ such that $\Theta_{3}^{2}\left(z_{3}\right)=q_{3}$. We modify the labeling $\Theta^{2}$ on the ball $B_{s_{3}}\left(G_{f(3)}, z_{3}\right)$. Let $\mathcal{A}^{2}=\left\{A_{i}^{2}\right\}_{i=1}^{\tau_{2}}$ be the set of all $\mathcal{F}_{2}$-labeled $G_{f(2)}$-balls of radius $s_{2}$ in $\Theta^{2}$ up to isomorphism. Let $\left\{w_{i}^{2}\right\}_{i=1}^{\tau_{2}} \subset B_{s_{3}}\left(G_{f(3)}, z_{3}\right) \cap\left(\Theta_{2}^{2}\right)^{-1}\left(q_{2}\right)$ be a subset of vertices such that:

- For any $1 \leq i \leq \tau_{2}$,

$$
\frac{1}{3} s_{3} \leq d_{G_{f(3)}}\left(z_{3}, w_{i}^{2}\right) \leq \frac{2}{3} s_{3} .
$$

- For any $1 \leq i \neq j \leq \tau_{2}$,

$$
d_{G_{f(3)}}\left(w_{i}^{2}, w_{j}^{2}\right)>20 r_{2} .
$$

Then for each $1 \leq i \leq \tau_{2}$ pick $z_{i}^{2} \in\left(\Theta_{2}^{2}\right)^{-1}\left(q_{2}\right)$ such that $B_{2 r_{2}}^{\Theta^{2}}\left(G_{f(2)}, z_{i}^{2}\right)$ fully contains an isomorphic copy of the ball $A_{i}^{2}$. Then using Proposition 4.2, for each $1 \leq i \leq \tau_{2}$ we simultaneously patch the balls $B_{3 r_{2}}^{\Theta^{2}}\left(G_{f(2)}, z_{i}^{2}\right)$ into $\Theta^{2}$ around $w_{i}^{2}$. Hence, we obtain a modified clean labeling $\hat{\Theta}^{3}: \Gamma \rightarrow \mathcal{F}$. So far, the construction of the 3-codeball was identical to the one of $B_{2}$. Now, we need some further considerations. It is possible that after the patching we will have some $g \in\left(\hat{\Theta}^{3}\right)^{-1}\left(q_{2}\right)$ such that the ball $B_{s_{2}}^{\hat{\Theta}^{3}, 2}\left(G_{f(2)}, g\right)$ is not isomorphic to the codeball $B_{2}$. Using Proposition 4.1, we patch the ball $B_{s_{2}+r_{1}}^{\Theta^{2}, 2}\left(G_{f(2)}, z_{2}\right)$ into $\hat{\Theta}^{3}$ around all such elements $g$ as above to obtain the clean labeling $\hat{\Theta}_{1}^{3}$. We call the $\mathcal{F}_{3}$-labeled $G_{f(3)}$-ball $B_{3}=B_{s_{3}}^{\hat{\Theta}_{1}^{3}, 3}\left(G_{f(3)}, z_{3}\right)$ the 3-codeball. Now we construct the clean labeling $\bar{\Theta}^{3}$ by patching the ball $B_{s_{3}+r_{2}}^{\hat{\Theta}_{1}^{3}, 3}\left(G_{f(3)}, z_{3}\right)$ into $\Theta^{2}$ around all vertices $y \in\left(\Theta_{3}^{2}\right)^{-1}\left(q_{3}\right)$. Again, due to the patchings, it is possible that for some $g \in\left(\bar{\Theta}^{3}\right)^{-1}\left(q_{2}\right), B_{s_{2}}^{\bar{\Theta}^{3}, 2}\left(G_{f(2)}, g\right)$ is not isomorphic to the 2-codeball $B_{2}$. We obtain the clean labeling $\Theta^{3}$ by patching the ball $B_{s_{2}+r_{1}}^{\Theta^{2}, 2}\left(G_{f(2)}, z_{2}\right)$ into $\bar{\Theta}^{3}$ around the vertices $g$ above. Then

- For all $z \in\left(\Theta_{3}^{3}\right)^{-1}\left(q_{3}\right)$ we have $B_{s_{3}, 3}^{\Theta^{3}, 3}\left(G_{f(3)}, z\right) \cong B_{3}$.

- For all $z \in\left(\Theta_{2}^{3}\right)^{-1}\left(q_{2}\right)$ we have $B_{s_{2}}^{\Theta^{3}, 2}\left(G_{f(2)}, z\right) \cong B_{2}$. 


\section{Minimal Cantor labelings III. (the induction)}

Let us suppose that we have already constructed the clean labelings $\Theta^{1}, \Theta^{2}, \ldots, \Theta^{n}$ satisfying the following properties.

- If $j \leq k \leq n$ and $\left(\Theta_{j}^{k}\right)(z)=q_{j}$, then the $\mathcal{F}_{j}$-labeled $G_{f(j)}$-ball $B_{s_{j}}^{\Theta^{k}, j}\left(G_{f(j)}, z\right)$ is isomorphic to a given $\mathcal{F}_{j}$-labeled $G_{f(j)}$-ball $B_{j}$, the j-codeball.

- For $1<j \leq n$, let $\mathcal{A}^{j-1}=\left\{A_{i}^{j-1}\right\}_{i=1}^{\tau_{j-1}}$ be the set of all $\mathcal{F}_{j-1}$-labeled $G_{f(j-1)}$-balls of radius $s_{j-1}$ in $\Theta^{j-1}$ up to isomorphism. Then for any $1 \leq i \leq \tau_{j-1}$, the $j$-codeball $B_{j}$ fully contains a copy of $A_{i}^{j-1}$.

Now we construct the clean labeling $\Theta^{n+1}$ in two rounds.

Round one: The $n+1$-codeball. Pick $z_{n+1} \in \Gamma$ such that $\Theta_{n+1}^{n}\left(z_{n+1}\right)=$ $q_{n+1}$. We modify the labeling $\Theta^{n}$ on the ball $B_{s_{n+1}}\left(G_{f(n+1)}, z_{n+1}\right)$. Let $\mathcal{A}^{n}=$ $\left\{A_{i}^{n}\right\}_{i=1}^{\tau_{n}}$ be the set of all $\mathcal{F}_{n}$-labeled $G_{f(n)}$-balls of radius $s_{n}$ in $\Theta^{n}$ up to isomorphism. Let $\left\{w_{i}^{n}\right\}_{i=1}^{\tau_{n}} \subset B_{s_{n+1}}\left(G_{f(n+1)}, z_{n+1}\right) \cap\left(\Theta_{n}^{n}\right)^{-1}\left(q_{n}\right)$ be a subset of vertices such that

- For any $1 \leq i \leq \tau_{n}$,

$$
\frac{1}{3} s_{n+1} \leq d_{G_{f(n+1)}}\left(z_{n+1}, w_{i}^{n}\right) \leq \frac{2}{3} s_{n+1} .
$$

- For any $1 \leq i \neq j \leq \tau_{n}$,

$$
d_{G_{f(n+1)}}\left(w_{i}^{n}, w_{j}^{n}\right)>20 r_{n} .
$$

By Rule 1, such system indeed exists. Now, for each $1 \leq i \leq \tau_{n}$ pick $z_{i}^{n} \in\left(\Theta_{n}^{n}\right)^{-1}\left(q_{n}\right)$ such that $B_{2 r_{n}}^{\Theta^{n}, n}\left(G_{f(n)}, z_{i}^{n}\right)$ fully contains an isomorphic copy of the ball $A_{i}^{n}$. Finally, using Proposition 4.2 , for each $1 \leq i \leq \tau_{n}$ we simultaneously patch the balls $B_{3 r_{n}}^{\Theta_{n}, n}\left(G_{f(n)}, z_{i}^{n}\right)$ into $\Theta^{n}$ around $w_{i}^{n}$. Hence, we obtain a modified clean labeling $\hat{\Theta}^{n+1}: \Gamma \rightarrow \mathcal{F}$. Now we need a definition to describe our procedure. Let $\Theta^{\prime}: \Gamma \rightarrow \mathcal{F}$ be a clean labeling and let $2 \leq i \leq n$. Let $\operatorname{Bad}_{i}\left(\Theta^{\prime}\right)$ be the set of all $y \in \Gamma$ such that $\Theta_{i}^{\prime}(y)=q_{i}$ and $B_{s_{i}}^{\Theta^{\prime}}, i\left(G_{f(i)}, y\right)$ is not isomorphic to the $i$-codeball $B_{i}$. The $i$-repair of $\Theta^{\prime}, \Theta_{<i>}^{\prime}$ is constructed in the following way. Using Proposition 4.1, we simultaneously patch the $\mathcal{F}_{i}$ labeled $G_{f(i)}$-ball $B_{s_{i}+r_{i-1}}^{\Theta^{i}, i}\left(G_{f(i)}, z_{i}\right)$ into $\Theta^{\prime}$ around all elements $y \in \operatorname{Bad}_{i}\left(\Theta^{\prime}\right)$. Here, $z_{i} \in \Gamma$ is an element for which $B_{s_{i}}^{\Theta^{i}, i}\left(G_{f(i)}, z_{i}\right)$ is isomorphic to the $i$ codeball $B_{i}$. Clearly, $\operatorname{Bad}_{i}\left(\Theta_{<i>}^{\prime}\right)$ is empty. Note however, that if $\operatorname{Bad}_{j}\left(\Theta^{\prime}\right)$ is empty for some $j<i$, it is possible that $\operatorname{Bad}_{j}\left(\Theta_{<i>}^{\prime}\right)$ is non-empty. Now, we construct inductively the clean labelings $\left\{\hat{\Theta}^{n+1, i}\right\}_{i=2}^{n+1}$. Let $\hat{\Theta}^{n+1, n+1}=\hat{\Theta}^{n+1}$ and for $2 \leq i \leq n$, let $\hat{\Theta}^{n+1, i}=\hat{\Theta}_{<i>}^{n+1, i+1}$.

Definition 6.1. Let $2 \leq i \leq n$. Let $i>j_{l}>j_{l-1}>\cdots>j_{1} \geq 2$ be integers and for $1 \leq k \leq l$ let $g_{k} \in \Gamma$ such that if $k<l$ then the set

$$
B_{s_{j_{k}}+5 r_{j_{k}-1}}\left(G_{f\left(j_{k}\right)}, g_{k}\right) \cap B_{s_{j_{k+1}}+5 r_{j_{k+1}-1}}\left(G_{f\left(j_{k+1}\right)}, g_{k+1}\right)
$$


is non-empty. Then, the set $\mathcal{L}=\cup_{k=1}^{l} B_{s_{j_{k}}+5 r_{j_{k}-1}}\left(G_{f\left(j_{k}\right)}, g_{k}\right)$ is called an $i$-chain of length $l$.

Lemma 6.1. The $G_{f(i)}$-diameter of an $i$-chain $\mathcal{L}$ is less than $\frac{1}{10} r_{i-1}$.

Proof. We have that

$$
\operatorname{diam}_{G_{f(i)}}(\mathcal{L}) \leq \sum_{k=1}^{l} \operatorname{diam}_{G_{f(i)}}\left(B_{s_{j_{k}}+5 r_{j_{k}-1}}\left(G_{f\left(j_{k}\right)}, g_{k}\right)\right)
$$

hence the lemma follows from Rule 2 .

Proposition 6.1. For any $2 \leq i \leq n, \operatorname{Bad}_{i}\left(\hat{\Theta}^{n+1,2}\right)$ is empty.

Proof. Let $\hat{\Theta}_{i}^{n+1, i}(w)=q_{i}$. It is enough to prove that $B_{s_{i}}^{\hat{\Theta}^{n+1,2}, i}\left(G_{f(i)}, w\right) \cong B_{i}$. Suppose that the ball $B_{s_{i}}^{\hat{\Theta}^{n+1,2}, i}\left(G_{f(i)}, w\right)$ is not isomorphic to the $i$-codeball $B_{i}$. Since $B_{s_{i}}^{\hat{\Theta}^{n+1, i}, i}\left(G_{f(i)}, w\right) \cong B_{i}$, there exists $h \in \Gamma$ and $j<i$ such that

- $h \in \operatorname{Bad}_{s_{j}}\left(\hat{\Theta}^{n+1, j+1}\right)$

- $B_{s_{j}+5 r_{j-1}}\left(G_{f(j)}, h\right) \cap B_{s_{i}}\left(G_{f(i)}, w\right) \neq 0$.

Let $l$ be the largest integer such that we have an $i$-chain $\mathcal{L}$ of length $l$ satisfying the following properties.

- $\mathcal{L} \cap B_{s_{i}}\left(G_{f(i)}, w\right) \neq 0$.

- $g_{k} \in \operatorname{Bad}_{s_{j_{k}}}\left(\hat{\Theta}^{n+1, j_{k}+1}\right)$, where

$$
\mathcal{L}=\cup_{k=1}^{l} B_{s_{j_{k}}+5 r_{j_{k}-1}}\left(G_{f\left(j_{k}\right)}, g_{k}\right) .
$$

So, by our previous observation we have that $l \geq 1$.

Lemma 6.2. The set

$$
B_{s_{j_{l}}+5 r_{j_{l}-1}}\left(G_{f\left(j_{l}\right)}, g_{l}\right) \cap\left(\Gamma \backslash B_{s_{i}+\frac{1}{2} r_{i-1}}\left(G_{f(i)}, w\right)\right)
$$

is nonempty.

Proof. If $B_{s_{j_{l}}+5 r_{j_{l}-1}}\left(G_{f\left(j_{l}\right)}, g_{l}\right)$ is contained in the ball $B_{s_{i}+\frac{1}{2} r_{i-1}}\left(G_{f(i)}, w\right)$, then $B_{s_{j_{l}}}^{\hat{\Theta}^{n+1, i}, j_{l}}\left(G_{f\left(j_{l}\right)}, g_{l}\right)$ is isomorphic to the $j_{l}$-codeball $B_{j_{l}}$. Thus, there is some $j_{l}<k<i$ and $g \in \Gamma$ such that $g \in \operatorname{Bad}_{k}\left(\hat{\Theta}^{n+1, k+1}\right)$ and the ball $B_{s_{k}+5 r_{k-1}}\left(G_{f(k)}, g\right)$ intersects the ball $B_{s_{j_{l}}+5 r_{j_{l}-1}}\left(G_{f\left(j_{l}\right)}, g_{l}\right)$, in contradiction with the maximality of $l$. Hence the lemma follows.

By Lemma 6.1, $\operatorname{diam}_{G_{f(i)}}(\mathcal{L}) \leq \frac{1}{10} r_{i-1}$. Therefore, it is not possible that both $\mathcal{L} \cap B_{s_{i}}\left(G_{f(i)}, w\right) \neq \emptyset$ and $\mathcal{L} \cap\left(\Gamma \backslash B_{s_{i}+\frac{1}{2} r_{i-1}}\left(G_{f(i)}, w\right)\right) \neq \emptyset$ hold. Therefore, our proposition follows.

We call the $\mathcal{F}_{n+1}$-labeled $G_{f(n+1)}$-ball $B_{n+1}=B_{s_{n+1}}^{\hat{\Theta}^{n+1,2}, n+1}\left(G_{f(n+1)}, z_{n+1}\right)$ the $n+1$-codeball. Now we construct the clean labeling $\bar{\Theta}^{n+1}$ by patching the ball $B_{s_{n+1}+r_{n}}^{\hat{\Theta}^{n+1,2}, n+1}\left(G_{f(n+1)}, z_{n+1}\right)$ into $\Theta^{n}$ around all the vertices $y \in\left(\Theta_{n+1}^{n}\right)^{-1}\left(q_{n+1}\right)$. 
Round two: The construction of $\Theta^{n+1}$. Again, it is possible that for some $2 \leq i \leq n$ the set $\operatorname{Bad}_{i}\left(\bar{\Theta}^{n+1}\right)$ is non-empty. Now, we inductively construct the clean labelings $\left\{\bar{\Theta}^{n+1, i}\right\}_{i=2}^{n+1}$. Let $\bar{\Theta}^{n+1, n+1}=\bar{\Theta}^{n+1}$ and for $2 \leq i \leq n$, let $\bar{\Theta}^{n+1, i}=\bar{\Theta}_{<i>}^{n+1, i+1}$. Copying the proof of Proposition 6.1, we can prove the following proposition.

Proposition 6.2. For any $2 \leq i \leq n, \operatorname{Bad}_{i}\left(\bar{\Theta}^{n+1,2}\right)$ is empty.

Let $\Theta^{n+1}=\bar{\Theta}^{n+1,2}$. Let us summarize what we have already proved about the clean labeling $\Theta^{n+1}$.

\section{Proposition 6.3.}

- For all $1<j \leq n+1$ and $z \in\left(\Theta_{j}^{n+1}\right)^{-1}\left(q_{j}\right)$ we have $B_{s_{j}}^{\Theta^{n+1}, j}\left(G_{f(j)}, z\right) \cong$ $B_{j}$.

- For any $1<j \leq n+1$ and $1 \leq i \leq \tau_{j-1}$, the $j$-codeball $B_{j}$ fully contains a copy of the ball $A_{i}^{j-1}$.

Finally, we have the following lemma.

Lemma 6.3. Let $z \in \Gamma$ such that $\Theta^{n+1}(z) \neq \Theta^{n}(z)$. Then $d_{G_{f(n+1)}}\left(z, e_{\Gamma}\right) \geq$ $5 s_{n+1}$.

Proof. By our construction if $\Theta^{n+1}(z) \neq \Theta^{n}(z)$ then there exists some $y \in \Gamma$ such that

- $\Theta_{n+1}^{n}(y)=q_{n+1}$ and there is an $l$-chain $\mathcal{L}$ for some $l \leq n$, intersecting $B_{2 s_{n+1}}\left(G_{f(n+1)}, y\right)$ for which $z \in B_{2 s_{n+1}}\left(G_{f(n+1)}, y\right) \cup \overline{\mathcal{L}}$.

Therefore, $z \in B_{5 s_{n+1}}\left(G_{f(n+1)}, y\right)$, hence the lemma follows from the definition of the clean labelings.

\section{Explicit COnstruction of a Free, minimal action}

Let $\left\{\Theta^{n}\right\}_{n=1}^{\infty}$ be the clean labelings constructed in the previous sections. By Lemma 6.3, for any $y \in \Gamma$ there exists $n_{y}>0$ such that if $n \geq n_{y}$, then $\Theta^{n}(y)=\Theta^{n_{y}}(y)$. Hence, $\lim _{n \rightarrow \infty} \Theta^{n}(y)=\Theta^{\infty}(y)$ is a well-defined clean labeling.

Lemma 7.1. For any $i \geq 2$ and $z \in\left(\Theta_{i}^{\infty}\right)^{-1}\left(q_{i}\right), B_{s_{i}}^{\Theta^{\infty}, i}\left(G_{f(i)}, z\right) \cong B_{i}$.

Proof. Let $n \geq i$ such that $\Theta^{\infty}(y)=\Theta^{n}(y)$ for all $y \in B_{s_{i}}\left(G_{f(i)}, z\right)$. Then, $\left(\Theta_{i}^{n}\right)(z)=q_{i}$. By Proposition 6.3, $B_{s_{i}}^{\Theta^{n}, i}\left(G_{f(i)}, z\right) \cong B_{i}$. Hence our lemma follows.

Lemma 7.2. Let $z \in \Gamma$, and $t, j \geq 1$ be integers. Then, there exists some $\rho>0$ such that the $\mathcal{F}_{j}$-labeled ball $B_{t}^{\Theta^{\infty}, j}\left(G_{f(j)}, z\right)$ is $(\rho, j)$-syndetic in $\Theta^{\infty}$. 
Proof. Let $q>j$ be an integer such that the $\mathcal{F}_{j}$-labeled ball $B_{t}^{\Theta^{m}, j}\left(G_{f(j)}, z\right)$ is isomorphic to $B_{t}^{\Theta^{\infty}, j}\left(G_{f(j)}, z\right)$, whenever $m \geq q$. Let $n>q$ be an integer such that $s_{n}>t$. Then the $n+1$-codeball $B_{n+1}$ contains a copy of the $\mathcal{F}_{n}$-labeled $G_{f(n)}$-ball $B_{s_{n}}^{\Theta^{n}, n}\left(G_{f(n)}, z\right)$. In particular, $B_{n+1}$ contains a copy of the ball $B_{t}^{\Theta^{n}, j}\left(G_{f(j)}, z\right) \cong B_{t}^{\Theta^{\infty}, j}\left(G_{f(j)}, z\right)$. The set $\left(\Theta_{1}^{\infty}\right)^{-1}\left(q_{1}\right)$ is a maximal $r_{1}$-sparse set, hence an $r_{1}$-net. The set $\left(\Theta_{2}^{\infty}\right)^{-1}\left(q_{2}\right)$ is a $\left(\Theta_{1}^{\infty}\right)^{-1}\left(q_{1}\right)$ maximal $r_{2}$-sparse set, hence by Lemma 4.1 an $r_{2}+r_{1}$-net. Inductively, $\left(\Theta_{n+1}^{\infty}\right)^{-1}\left(q_{n+1}\right)$ is a $\sum_{i=1}^{n+1} r_{i}$-net, thus a $2 r_{n+1}$-net. So, for every $x \in \Gamma$ the ball $B_{3 r_{n+1}, n+1}^{\Theta^{\infty}}\left(G_{f(n+1)}, x\right)$ contains a copy of $B_{t}^{\Theta^{\infty}, j}\left(G_{f(j)}, z\right)$. That is, the $\mathcal{F}_{j}$-labeled $G_{f(j)}$-ball $B_{t}^{\Theta^{\infty}, j}\left(G_{f(j)}, z\right)$ is $\left(3 r_{n+1}, j\right)$-syndetic in $\Theta^{\infty}$.

Theorem 5. The orbit closure of $\Theta^{\infty}$ in the Bernoulli shift space $\mathcal{F}^{\Gamma}$ is free and minimal.

By Lemma 7.2, the orbit closure of $\Theta^{\infty}$ is minimal. Since $\Theta^{\infty}$ is a clean labeling, it is proper in the sense that for any $r>0$ there exists some $s_{r}>0$ such that if $0<d_{G_{r}}(x, y) \leq r$, then

$$
\Theta_{\left[s_{r}\right]}^{\infty}(x) \neq \Theta_{\left[s_{r}\right]}^{\infty}(y) .
$$

Therefore, by repeating the argument of the proof of Proposition 1.1, we immediately obtain that the action of $\Gamma$ on the orbit closure of $\Theta^{\infty}$ is free.

\section{THE DYNAMICAL VERSION OF CLEAN LABELINGS}

In this section we prove some propositions and lemmas that we need for the proof of Theorem 2. For the whole section let the integers $\left\{s_{i}\right\}_{i=1}^{\infty},\left\{r_{i}\right\}_{i=1}^{\infty}$ and the finite sets $\left\{F_{m}\right\}_{m=1}^{\infty}$ be as in the previous sections. Our first proposition is about the existence of the dynamical analogue of clean labelings.

Proposition 8.1. Let $\alpha: \Gamma \curvearrowright \mathcal{C}$ be a free continuous action as in the previous sections. Then there exists a continuous map

$$
\Sigma=\Sigma_{\mathcal{C}} \times \prod_{m=1}^{\infty} \Sigma_{m}: \mathcal{C} \rightarrow \mathcal{F}
$$

such that for any $x \in \mathcal{C}, \Sigma_{x}(\gamma):=\Sigma(\alpha(\gamma)(x))$ defines an almost clean labeling of the group $\Gamma$ (see Section 4). We call $\Sigma$ a dynamical clean labeling.

Proof. Before starting the proof let us fix some definitions and notations. As in Section 2, we will consider the Borel graphs $\mathcal{G}_{r}$ on the Cantor set $\mathcal{C}$ associated to the free action $\alpha$. If $U$ is a clopen subset of $\mathcal{C}$, then we define a subset of our countable group $\Gamma$ by

$$
O_{x}^{U}:=\{\gamma \in \Gamma \mid \alpha(\gamma)(x) \in U\} .
$$

Let $t>0$ be an integer. We call a clopen set $U \subset \mathcal{C} t$-separated in the Borel graph $\mathcal{G}_{r}$ if for any $x \neq y \in U, d_{\mathcal{G}_{r}}(x, y)>t$. Clearly, $U$ is $t$-separated if and only if for any $x \in \mathcal{C}$ the set $O_{x}^{U}$ is $t$-sparse in the graph $G_{r}$. 
Lemma 8.1. Let $U \subset \mathcal{C}$ be a clopen set and $t>0$ be an integer. Then there exists a clopen set $V \subset \mathcal{C}$ such that for any $x \in \mathcal{C}$, the set $O_{x}^{V}$ is an $O_{x}^{U}$-maximal t-sparse set in the graph $G_{r}$.

Proof. By continunity and freeness, there exists some $s>0$ such that if for some $x, y \in \mathcal{C}$ we have $0<d_{\mathcal{G}_{r}}(x, y) \leq t$, then $(x)_{s} \neq(y)_{s}$. Let $a_{1}, a_{2}, \ldots, a_{2^{s}}$ be an enumeration of the set $\{0,1\}^{\{1,2, \ldots, s\}}$. For $1 \leq i \leq 2^{s}$, let

$$
W_{i}:=\left\{x \in \mathcal{C} \mid(x)_{s}=a_{i}\right\} .
$$

Now let $V_{1}:=U \cap W_{1}$ and

$$
Z_{1}=\cup_{d_{G_{r}}\left(\gamma, e_{\Gamma}\right) \leq t} \alpha(\gamma) V_{1} .
$$

Clearly, $Z_{1}$ is a clopen set. Let $Z_{1}^{c}$ denote the complement of $Z_{1}$. Now, let $V_{2}:=W_{2} \cap U \cap Z_{1}^{c}$. Then for any $x \in \mathcal{C}, O_{x}^{V_{1} \cup V_{2}}$ is a $t$-sparse set in $G_{r}$ contained in the set $O_{x}^{U}$. Inductively, suppose that for $1 \leq i \leq n<2^{s}$,

- $V_{i} \subset W_{i} \cap U$ is a clopen set,

- $Z_{i}=\cup_{d_{G_{r}}\left(\gamma, e_{\Gamma}\right) \leq t} \alpha(\gamma)\left(\cup_{j=1}^{i} V_{j}\right)$,

- $V_{i+1}=W_{i+1} \cap U \cap Z_{i}^{c}$.

Then for any $1 \leq i \leq n, O_{x}^{\cup_{j=1}^{i} V_{j}}$ is a $t$-sparse set in the graph $G_{r}$ which is contained in the set $O_{x}^{U}$. We define

$$
V_{n+1}:=W_{n+1} \cap U \cap Z_{n}^{c} .
$$

Then for any $x \in \mathcal{C}, O_{x}^{\cup_{j=1}^{n+1} V_{j}} \subset O_{x}^{U}$ is a $t$-sparse set in the graph $G_{r}$. We claim that for any $x \in \mathcal{C}, O_{x}^{\cup_{j=1}^{2^{s}} V_{j}}$ is an $O_{x}^{U}$-maximal $t$-sparse set in $G_{r}$. Clearly, $O_{x}^{\cup_{j=1}^{s} V_{j}} \subset O_{x}^{U}$ and $O_{x}^{\cup_{j=1}^{2 s} V_{j}}$ is a $t$-sparse set in the graph $G_{r}$. Suppose that $O_{x}^{\cup_{j}^{2 s} V_{j}}$ is not an $O_{x}^{U}$-maximal $t$-sparse set in the graph $G_{r}$. Then there exists $\delta \in \Gamma_{r}$ such that $\alpha(\delta)(x) \in U$ and

$$
d_{G_{r}}\left(\delta, O_{x}^{\cup_{j=1}^{s} V_{j}}\right)>t .
$$

Let $(\alpha(\delta)(x))_{s}=a_{i}$. Then by $(1)$,

$$
\alpha(\delta)(x) \in W_{i} \cap U \cap Z_{i-1}^{c}=V_{i}
$$

in contradiction with our assumption.

Lemma 8.2. Let $V$ be an $r_{m}$-separated clopen set in the Borel graph $\mathcal{G}_{f(m)}$. Then there exists a continuous function $\varphi: \mathcal{C} \rightarrow F_{m}$ such that

- $V=\varphi^{-1}\left(q_{m}\right)$.

- For any $q \in F_{m}$, the clopen set $\varphi^{-1}(q)$ is $r_{m}$-separated in the Borel graph $\mathcal{G}_{f(m)}$.

Proof. Let $V_{1}:=V$. Using Lemma 8.1, we pick $V_{2}$ in such a way that for any $x \in \mathcal{C}, O_{x}^{V_{2}}$ is an $O_{x}^{V_{1}^{c}}$-maximal $r_{m}$-sparse set in the graph $G_{f(2)}$. Inductively, suppose that the clopen sets $V_{1}, V_{2}, \ldots, V_{i}$ have already been picked. Then, 
let $V_{i+1} \subset \mathcal{C}$ be a clopen set such that for any $x \in \mathcal{C}, O_{x}^{V_{i+1}}$ is an $O_{x}^{\left(\cup_{j=1}^{i} V_{j}\right)^{c}}$. maximal $r_{m}$-sparse set in the graph $G_{f(m)}$. It is enough to show that $\cup_{i=1}^{\left|F_{m}\right|} V_{i}=$ $\mathcal{C}$. Suppose that $x \in \mathcal{C} \backslash \cup_{i=1}^{\left|F_{m}\right|} V_{i}$. By Rule 3, there exists $1 \leq i \leq\left|F_{m}\right|$, such that for any $\gamma$ for which $d_{G_{f(m)}}\left(\gamma, e_{\Gamma}\right) \leq r_{m}$ holds, we have that $\alpha(\gamma)(x) \notin V_{i}$. However, this is in contradiction with the definition of the set $V_{i}$.

Now we finish the proof of our proposition. First, using Lemmas 8.1 and 8.2 we construct a continuous map $\Sigma_{1}: \mathcal{C} \rightarrow F_{1}$ such that for any $q \in F_{1}$ the set $\left(\Sigma_{1}\right)^{-1}(q)$ is $r_{1}$-separated in the Borel graph $\mathcal{G}_{f(1)}$. Then, using the lemmas we construct $\Sigma_{2}: \mathcal{C} \rightarrow F_{2}$ such that for any $q \in F_{2},\left(\Sigma_{2}\right)^{-1}(q)$ is $r_{2}$-separated in $\mathcal{G}_{f(2)}$ and for any $x \in \mathcal{C}, O_{x}^{\left(\Sigma_{2}\right)^{-1}\left(q_{2}\right)}$ is a $\left(\Sigma_{1}\right)^{-1}\left(q_{1}\right)$-maximal $r_{2}$-sparse set in the graph $G_{f(2)}$. Inductively, we construct $\Sigma_{m}$ for all $m \geq 1$.

Remark: The inductive part in the proof of the previous proposition is the reason that in the definition of clean labelings we required $\left(\Theta_{m}\right)^{-1}\left(q_{m}\right)$ to be a $\left(\Theta_{m-1}\right)^{-1}\left(q_{m-1}\right)$-maximal $r_{m}$-sparse subset instead of being a maximal $r_{m}$-sparse subset that is contained in $\left(\Theta_{m-1}\right)^{-1}\left(q_{m-1}\right)$.

The following two lemmas are straightforward consequences of the definitions.

Lemma 8.3. Let

$$
\Sigma^{\prime}=\Sigma_{\mathcal{C}}^{\prime} \times \prod_{m=1}^{\infty} \Sigma_{m}^{\prime}: \mathcal{C} \rightarrow \mathcal{F}
$$

and

$$
\Theta^{\prime}=\Theta_{\mathcal{C}}^{\prime} \times \prod_{m=1}^{\infty} \Theta_{m}^{\prime}: \mathcal{C} \rightarrow \mathcal{F}
$$

be continuous functions. Let $U \subset \mathcal{C}$ be a $2 t$-separated clopen set in the Borel graph $\mathcal{G}_{r}$. Also, let $B_{t}^{\Theta, i}\left(G_{r}, e_{\Gamma}\right)$ be a $\mathcal{F}_{i}$-labeled ball of radius $t$ in the graph $G_{r}$. Let

$$
\Sigma "=\Sigma "_{\mathcal{C}} \times \prod_{m=1}^{\infty} \Sigma{ }_{m}: \mathcal{C} \rightarrow \mathcal{C} \times \prod_{m=1}^{\infty} F_{m}
$$

be defined in the following way.

- If $d_{\mathcal{G}_{r}}(x, U)>t$, then $\Sigma "(x)=\Sigma^{\prime}(x)$.

- If there exists $y \in U$ and $\gamma \in \Gamma, d_{G_{r}}\left(\gamma, e_{\Gamma}\right) \leq t$ such that $\alpha(\gamma)(y)=x$, then let $\Sigma^{\prime \prime}{ }_{[i]}(x)=\Theta_{[i]}^{\prime}(\gamma),\left(\Sigma^{\prime \prime}{ }_{\mathcal{C}}(x)\right)_{i^{+}}=\left(\Sigma_{\mathcal{C}}^{\prime}(x)\right)_{i^{+}}$and for $j>i$, let $\Sigma^{\prime \prime}{ }_{j}(x)=\Sigma_{j}^{\prime}(x)$.

Then $\Sigma "$ is a continuous map as well.

Lemma 8.4. Let $V \subset \mathcal{C}$ be a t-separated clopen set. Let $s<t$ and suppose that for some $\varepsilon>0$ we have

$$
\left|B_{s}\left(G_{r}, e_{\Gamma}\right)\right| \leq \varepsilon\left|B_{t}\left(G_{r}, e_{\Gamma}\right)\right| .
$$


Let $U \subset \mathcal{C}$ be the set of elements $x$ such that there exists $y \in V$ so that $d_{\mathcal{G}_{r}}(x, y) \leq s$. Then, for any Borel probability measure $\mu$ on $\mathcal{C}$ that is invariant under the action $\alpha$,

$$
\mu(U)<\varepsilon \text {. }
$$

\section{The Proof of Theorem 2}

Let $\zeta: \Gamma \curvearrowright \mathcal{C}$ be a free continuous action. Let $\left\{\Sigma^{i}: \mathcal{C} \rightarrow \mathcal{F}\right\}_{i=1}^{\infty}$ be a sequence of dynamical clean labelings. We call $y \in \mathcal{C}$ stable (with respect to the system $\left.\left\{\Sigma^{i}\right\}_{i=1}^{\infty}\right)$ if for any element $x \in \mathcal{C}$ in the $\Gamma$-orbit of $y$, there exists $n_{x}>0$ such that if $n \geq n_{x}$ then $\Sigma^{n}(x)=\Sigma^{n_{x}}(x)$. So, for the $\Gamma$-invariant subset of stable points $y, \lim _{n \rightarrow \infty} \Sigma^{n}(y)=\Sigma^{\infty}(y)$ exists.

Proposition 9.1. There exists a sequence of dynamical clean labelings $\left\{\sum^{i}\right\}_{i=1}^{\infty}$ such that

- For any Borel probability measure $\mu$ that is invariant under the action $\zeta$, the $\mu$-measure of the stable points is 1 .

- There exists a free and minimal Bernoulli subshift $M \subset \mathcal{F}^{\Gamma}$ such that for each stable point $y \in \mathcal{C}$,

$$
\gamma \rightarrow \Sigma^{\infty}(\zeta(\gamma)(y))
$$

defines an injective equivariant Borel map from the Borel set of stable points to $M$.

Proof. We proceed very similarly as we did in the construction of $\Theta^{\infty}$. The following lemma is an immediate corollary of Lemma 8.3.

Lemma 9.1. Let

$$
\Sigma^{a}: \Sigma_{\mathcal{C}}^{a} \times \prod_{m=1}^{\infty} \Sigma_{m}^{a}: \mathcal{C} \rightarrow \mathcal{F}
$$

be a dynamical clean labeling and

$$
\Theta^{d}: \Theta_{\mathcal{C}}^{d} \times \prod_{m=1}^{\infty} \Theta_{m}^{d}: \Gamma \rightarrow \mathcal{F}
$$

be an almost clean labeling. Let $\Theta_{n}^{d}(x)=q_{n}$. Let $V \subset \mathcal{C}$ be an $r_{n}$-separated

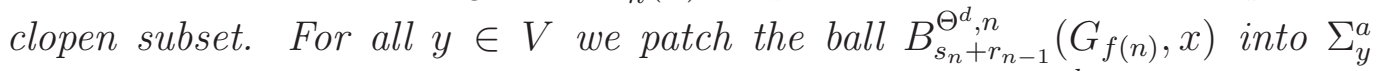
around $y$. Then we obtain a new dynamical clean labeling $\Sigma^{b}$.

The construction of $\Sigma^{2}$. Let $\Sigma^{1}$ be a dynamical clean labeling such that for $x=\left(x_{1}, x_{2}, x_{3}, \ldots\right) \in \mathcal{C}, \Sigma_{\mathcal{C}}^{1}(x)=\left(y_{1}, y_{2}, y_{3}, \ldots\right)$, where for $n \geq 0, y_{2^{n}}=x_{1}$ and if $2^{n}<a<2^{n+1}$, then $y_{a}=x_{a-2^{n}+1}$. Let $x \in \mathcal{C}$ and $\Theta^{1}:=\Sigma_{x}^{1}$. Let $\hat{\mathcal{A}}^{1}=\left\{\hat{A}_{i}^{1}\right\}_{i=1}^{\hat{r}_{1}}$ be the set of all $\mathcal{F}_{1}$-labeled $G_{f(1)}$-balls of radius $s_{1}$ in the almost clean labelings $\left\{\Sigma_{w}^{1}\right\}_{w \in \mathcal{C}}$. Note that $\hat{\mathcal{A}}^{1}$ might contain balls that are not in $\Theta^{1}$. Let $z_{2} \in\left(\Theta_{2}^{1}\right)^{-1}\left(q_{2}\right)$. Proceeding in the same way as in Section 5 (using $\hat{\mathcal{A}}^{1}$ instead of $\mathcal{A}^{1}$ ), we construct the modified labeling $\hat{\Theta}^{2}: \Gamma \rightarrow \mathcal{F}$. Now, 
our new 2-codeball will be $\hat{B}_{2}=B_{s_{2}}^{\hat{\Theta}^{2}, 2}\left(G_{f(2)}, z_{2}\right)$. Then, we patch the ball $B_{s_{2}+r_{1}}^{\hat{\Theta}^{2}, 2}\left(G_{f(2)}, z_{2}\right)$ into $\Sigma^{1}$ around all vertices $y \in\left(\Sigma^{1}\right)^{-1}\left(q_{2}\right)$ to obtain (Lemma 9.1) the dynamical clean labeling $\Sigma^{2}$.

The induction. Let us suppose that we have already constructed the dynamical clean labelings $\Sigma^{1}, \Sigma^{2}, \ldots, \Sigma^{n}$ satisfying the following properties.

- If $j \leq k \leq n, x \in \mathcal{C}, \Theta^{k}=\Sigma_{x}^{k}$ and $\left(\Theta_{j}^{k}\right)(z)=q_{j}$, then the $\mathcal{F}_{j}$-labeled $G_{f(j)}$-ball $B_{s_{j}}^{\Theta^{k}, j}\left(G_{f(j)}, z\right)$ is isomorphic to a given $\mathcal{F}_{j}$-labeled $G_{f(j)}$-ball $\hat{B}_{j}$, our new j-codeball.

- For $1<j \leq n$, let $\hat{\mathcal{A}}^{j-1}=\left\{\hat{A}_{i}^{j-1}\right\}_{i=1}^{\hat{\tau}_{j-1}}$ be the set of all $\mathcal{F}_{j-1}$-labeled $G_{f(j-1)}$-balls of radius $s_{j-1}$ in the almost clean labelings $\left\{\Sigma_{w}^{j-1}\right\}_{w \in \mathcal{C}}$ up to isomorphism. Then for any $1 \leq i \leq \hat{\tau}_{j-1}$, the $j$-codeball $\hat{B}_{j}$ fully contains a copy of $\hat{A}_{i}^{j-1}$.

Again, we construct the dynamical clean labeling $\sum^{n+1}$ in two rounds.

Round one: The new $n+1$-codeball. Let $x \in \mathcal{C}$ and $\Theta^{n}=\Sigma_{x}^{n}$. Also, let $z_{n+1} \in \Gamma$ such that $\Theta_{n+1}^{n}\left(z_{n+1}\right)=q_{n+1}$. Let $\hat{\mathcal{A}}^{n}=\left\{\hat{A}_{i}^{n}\right\}_{i=1}^{\hat{\tau}_{n}}$ be the set of all $\mathcal{F}_{n}$-labeled $G_{f(n)}$-balls of radius $s_{n}$ in the almost clean labelings $\left\{\sum_{w}^{n}\right\}_{w \in \mathcal{C}}$ up to isomorphism. Now we construct the almost clean labeling $\hat{\Theta}^{n+1,2}: \Gamma \rightarrow \mathcal{F}$ exactly the same way as in Section 6 . The $\mathcal{F}_{n+1}$-labeled $G_{f(n+1)}$-ball $\hat{B}_{n+1}=B_{s_{n+1}}^{\hat{\Theta}^{n+1,2}, n+1}\left(G_{f(n+1)}, z_{n+1}\right)$ will be the new $n+1$-codeball. Finally, we construct the dynamical clean labeling $\bar{\Sigma}^{n+1}$ by patching the ball $B_{s_{n+1}+r_{n}}^{\hat{\Theta}^{n+1,2}, n+1}\left(G_{f(n+1)}, z_{n+1}\right)$ into $\Sigma^{n}$ around all the vertices $y \in\left(\sum_{n+1}^{n}\right)^{-1}\left(q_{n+1}\right)$.

Round two: The construction of $\Sigma^{n+1}$. For a dynamical clean labeling $\Sigma^{\prime}$, the set $\operatorname{Bad}_{i}\left(\Sigma^{\prime}\right)$ is clopen so by Lemma 8.3 we can construct the dynamical clean labelings $\bar{\Sigma}^{n+1, n+1}, \bar{\Sigma}^{n+1, i}=\bar{\Sigma}_{<i>}^{n+1, i+1}$ in the same way as in Section 6 . Let $\Sigma^{n+1}=\bar{\Sigma}^{n+1,2}$ and let $Y \subset \mathcal{C}$ be the set of stable points with respect to the family of dynamical clean labelings $\left\{\Sigma^{i}\right\}_{i=1}^{\infty}$. Then $\Sigma^{\infty}(y)=\lim _{n \rightarrow \infty} \Sigma^{n}(y)$ is a well-defined Borel map on the invariant Borel set $Y$. By Theorem 5, for any $y \in Y$, the orbit closure of $\Theta_{y}^{\infty}$ in the Bernoulli shift space $\mathcal{F}^{\Gamma}$ is free and minimal. By our construction of the codeballs, if $y_{1}, y_{2} \in Y$, then for any $i \geq 1$, the set of $\mathcal{F}_{i}$-labeled $G_{f(i)}$-balls in $\Theta_{y_{1}}^{\infty}$ and $\Theta_{y_{2}}^{\infty}$ coincide. Hence the orbit closures of $\Theta_{y_{1}}^{\infty}$ and $\Theta_{y_{2}}^{\infty}$ coincide as well. So, let $M$ be the orbit closure of $\Theta_{y}^{\infty}$ in $\mathcal{F}^{\Gamma}$, where $y \in Y$. Notice that the map $\Phi: Y \rightarrow M$ defined by $\Phi(y)(\gamma)=\Sigma^{\infty}(\zeta(\gamma)(y))$ is injective. Indeed, if $y_{1} \neq y_{2} \in Y$, then $\Sigma_{\mathcal{C}}^{1}\left(y_{1}\right)$ and $\Sigma_{\mathcal{C}}^{1}\left(y_{2}\right)$ differ from each other in infinitely many digits. Hence, $\Sigma^{\infty}\left(y_{1}\right) \neq \Sigma^{\infty}\left(y_{2}\right)$. We finish the proof of our proposition by showing that for any Borel probability measure $\mu$ invariant under the action $\zeta, \mu(Y)=1$. Let $Q_{n} \subset \mathcal{C}$ be the set of elements $w$ such that $\Sigma^{n+1}(w) \neq \Sigma^{n}(w)$. By the 
Borel-Cantelli Lemma, it is enough to prove that

$$
\sum_{n=1}^{\infty} \mu\left(Q_{n}\right)<\infty .
$$

Let $z \in Q_{n}$ and let $V=\left(\sum_{n+1}^{n}\right)^{-1}\left(q_{n+1}\right)$. As we observed in the proof of Lemma 6.3 , there exists $y \in V$ and $\gamma \in B_{5 s_{n+1}}\left(G_{f(n+1)}, e_{\Gamma}\right)$ such that $\zeta(\gamma)(y)=z$. Note that $V$ is a $\frac{1}{10} r_{n+1}$-separated clopen set and by Rule 2 ,

$$
\left|B_{\frac{1}{10} r_{n+1}}\left(G_{f(n+1)}, e_{\Gamma}\right)\right| \geq 10^{n+1}\left|B_{5 s_{n+1}}\left(G_{f(n+1)}, e_{\Gamma}\right)\right| .
$$

Thus by Lemma 8.4 we have that $\mu\left(Q_{n}\right)<10^{-(n+1)}$, therefore $(2)$ holds. This finishes the proof of our proposition.

Now we can finish the proof of Theorem 2. Let $\zeta: \Gamma \curvearrowright \mathcal{C}$ be the universal action of Theorem 3. Let $\alpha: \Gamma \curvearrowright M$ be the Bernoulli subshift and $\left\{\Sigma^{i}\right\}_{i=1}^{\infty}$ be the sequence of dynamical clean labelings in Proposition 9.1. Let $\beta: \Gamma \curvearrowright$ $X$ be a Borel action, let $\nu$ be a Borel probability measure on $X$ invariant under $\beta$, and finally let $\Psi_{\beta}: X \rightarrow \mathcal{C}$ be the equivariant map in Theorem 3 . Then $\left(\Psi_{\beta}\right)_{*}(\nu)$ is a Borel probability measure invariant under the action $\zeta$. Let $Y \subset \mathcal{C}$ be the set of stable points with respect to the system $\left\{\Sigma^{i}\right\}_{i=1}^{\infty}$. Then, $\Phi(y)(\gamma)=\Sigma^{\infty}(\zeta(\gamma)(y))$ defines an injective, equivariant Borel map $\Phi: Y \rightarrow M$. Let $X^{\prime}=\left(\Psi_{\beta}\right)^{-1}(Y)$. Then by Proposition 9.1, $\nu\left(X^{\prime}\right)=1$ and $\Phi \circ \Psi_{\beta}: X^{\prime} \rightarrow M$ is an injective, equivariant Borel map. Hence, our theorem follows.

Finally, we prove Corollary 1.1. Let $\beta: \Gamma \curvearrowright \mathcal{C}$ be the action in Theorem 2. First let us suppose that $\Gamma$ is amenable. Let $\alpha: \Gamma \curvearrowright(X, \mu)$ be an ergodic action and $\Phi_{\alpha}\left(X^{\prime}\right) \rightarrow \mathcal{C}$ be an equivariant injective map. Then the KolmogorovSinai entropies of $\alpha$ and $\left(\Phi_{\alpha}\right)_{*}(\mu)$ coincide. Also, $\left(\Phi_{\alpha}\right)_{*}(\mu)$ is ergodic. Since for any $0<c \leq \infty$ we have an ergodic action with Kolmogorov-Sinai entropy $c$, our corollary follows for amenable groups. Now let us suppose that $\Gamma$ is non-amenable. Again, we need to prove that there exist more than one non-isomorphic essentially free ergodic actions of $\Gamma$. However, by the famous theorem of Epstein every non-amenable group has uncountably many pairwise orbit-inequivalent actions [4] so our corollary follows for non-amenable groups as well.

\section{REFERENCES}

[1] M. AbÉRT And G. ElEk, Hyperfinite actions on countable sets and probability measure spaces. Dynamical systems and group actions, 1-16, Contemp. Math., 567, Amer. Math. Soc., Providence, RI, 2012.

[2] A. Bernshteyn, Free subshifts with invariant measures from the Lovász Local Lemma. preprint arXiv:1802.07123

[3] G. EleK, Uniformly recurrent subgroups and simple $C^{*}$-algebras. Journal of Funct. Anal. 274 (2018) no.6 1657-1689.

[4] I. EPstein, Orbit inequivalent actions of non-amenable groups. preprint: https://arxiv.org/abs/0707.4215 
[5] E. Glasner and B. Weiss, Uniformly recurrent subgroups. Recent trends in ergodic theory and dynamical systems Contemp. Math., 631, Amer. Math. Soc., Providence, RI, 2015.

[6] G. Huorth and M. Molberg, Free continuous actions on zero-dimensional spaces. Topology Appl. 153 (2006), no. 7, 1116-1131.

[7] A.S. Kechris, S. Solecki and S. Todorcevic, Borel chromatic numbers. Adv. Math. 141, (1999), 1-44.

[8] N. Matte Bon and T. Tsankov, Realizing uniformly recurrent subgroups preprint: arXiv: 1702.07101

[9] J. C. Охтовy, Ergodic sets. Bull. Amer. Math. Soc. 58, (1952), 116-136.

[10] B. Seward and R.D. Tucker-Drob, Borel structurability on the 2-shift of a countable group. Ann. Pure Appl. Logic, 167 (2016), no. 1, 1-21.

[11] B. Weiss, Minimal models for free actions. Dynamical systems and group actions, Contemp. Math 567, (2012), 249-264.

Department of Mathematics And Statistics, Fylde College, Lancaster UniVERSity, LANCASTER, LA1 4YF, United Kingdom

E-mail address: g.elek@lancaster.ac.uk 